Federal Reserve Bank of Minneapolis

Research Department

\title{
Optimal Monetary Impulse-Response Functions in a Matching Model
}

\author{
Brett Katzman, John Kennan, \\ and Neil Wallace*
}

Working Paper 595

September 1999

\begin{abstract}
The effects on ex ante optima of a lag in seeing monetary realizations are studied using a matching model of money. The main new ingredient in the model is meetings in which producers have more information than consumers. A consequence is that increases in the amount of money that occur with small enough probability can have negative impact effects on output, because it is optimal to shut down trade in such low probability meetings rather than have lower output when high probability realizations occur. The information lag also produces prices that do not respond much to current monetary realizations.
\end{abstract}

JEL classification numbers: E30, E40, D82

*Katzman, University of Miami; Kennan, University of Wisconsin, Madison and NBER; Wallace, Federal Reserve Bank of Minneapolis and The Pennsylvania State University. We thank Edward J. Green, Nobuhiro Kiyotaki, Narayana Kocherlakota, and Rody Manuelli for helpful comments. The views expressed herein are those of the authors and not necessarily those of the Federal Reserve Bank of Minneapolis or the Federal Reserve System. 


\title{
Optimal Monetary Impulse-Response Functions in a Matching Model* $^{*}$
}

\author{
Brett Katzman $\dagger$ John Kennan; and Neil Wallace ${ }^{\S}$
}

September 17, 1999

\begin{abstract}
The effects on ex ante optima of a lag in seeing monetary realizations are studied using a matching model of money. The main new ingredient in the model is meetings in which producers have more information than consumers. A consequence is that increases in the amount of money that occur with small enough probability can have negative impact effects on output, because it is optimal to shut down trade in such low probability meetings rather than have lower output when high probability realizations occur. The information lag also produces prices that do not respond much to current monetary realizations.
\end{abstract}

JEL classification \#'s: E30, E40, D82.

\section{Introduction}

We study the role of lags in seeing monetary realizations in a matching model of money. We do this by fixing the lag and varying the fraction of the population who know about the current realization without a lag. The background environment is a familiar discrete-time, random-matching model with divisible and perishable produced goods, indivisible money, and a unit upper bound on individual money holdings. The aggregate amount of money follows a finite-state, first-order Markov process. At each date, the population is divided in a random way into a group, called the informed, who see current realizations when they occur, and a group, called the uninformed, who see them with a one-period lag. In this setting, we study how ex ante optima depend on the persistence of the monetary uncertainty and on the size of the informed group. In particular, we describe how the optimal responses of total output and the price level to monetary realizations vary with those features of the environment. In studying an ex ante optimum, we are studying the output and price level responses

${ }^{*}$ We thank Edward J. Green, Nobuhiro Kiyotaki, Narayana Kocherlakota, and Rody Manuelli for helpful comments.

${ }^{\dagger}$ University of Miami.

$\ddagger$ University of Wisconsin, Madison and NBER.

$\S$ The Pennsylvania State University and the Federal Reserve Bank of Minneapolis. Please address communications regarding this paper to Neil Wallace, 608 Kern, The Pennsylvania State University, University Park, PA 16802-3306; e-mail:<neilw@psu.edu>. 
to monetary realizations for a society that cannot control its money supply process or the asymmetric information about it. The society optimizes by choosing the way trade will be conducted in meetings subject to individual rationality and truth-telling constraints.

Our model has three attractive features. One is its similarity to the world described by David Hume in his attempt to describe and explain the effects on output and prices of money-supply changes. He said,

...Accordingly we find, that, in every kingdom, into which money begins to flow in greater abundance than formerly, every thing takes a new face: labour and industry gain life; the merchant becomes more enterprising,...

To account, then, for this phenomenon, we must consider, that though the high price of commodities be a necessary consequence of the encrease of gold and silver, yet it follows not immediately upon that encrease; but some time is required before the money circulates through the whole state, and makes its effect be felt on all ranks of people. At first, no alteration is perceived; by degrees the price rises, first of one commodity, then of another; till the whole at last reaches a just proportion with the new quantity of specie which is in the kingdom. In my opinion, it is only in this interval or intermediate situation, between the acquisition of money and rise of prices, that the encreasing quantity of gold and silver is favorable to industry. When any quantity of money is imported into a nation, it is not at first dispersed into many hands but is confined to the coffers of a few persons, who immediately seek to employ it to advantage. ...([5] pages $37,38)$

There seem to be three explanatory ingredients in Hume's discussion: decentralized trade, asymmetric information about the quantity of money, and an initial distribution of money that differs from the steady-state distribution. Because our model contains versions of all three ingredients, it provides a nice testing ground for Hume's notion that the existence of uninformed people is important.

A second attractive feature of the model is that the existence of uninformed people is very natural in the matching model context. In fact, a necessary condition for money to be essential is that people not know what is happening in all other meetings; if they did, then they would know their current trading partner's history and that, in turn, would eliminate an essential role for money (see the discussions in Kocherlakota [9] and Wallace [16]). Not knowing what is happening in all other meetings leads directly to the existence of uninformed people. Indeed, the assumption that seems somewhat artificial is the public announcement of the state after one date. That assumption is adopted for convenience. Letting the public announcement occur with a greater lag is a natural extension, but one which comes at the price of a larger state space.

A third attractive feature, and, perhaps, the most important for the substantive results, is that our specification breaks the tight link between who knows and who produces that appears in most information-based models of output responses 
to money supply changes. In most such models, the producers are uninformed and the consumers are informed (see Lucas [10], Eden [4], Lucas and Woodford [11] and Wallace [15]). As noted in Barro and King [1], that information pattern tends to bias outcomes in favor of expansionary effects of money-supply increases and contractionary effects for decreases. In our setting, the informed are a random subset of the population, some of whom end up as producers and some as consumers. In having some meetings with relatively informed consumers and other meetings with relatively informed producers, our model resembles that of Jones and Manuelli [6]. There are, though, two main differences.

One is that Jones and Manuelli impose a bargaining rule, while we study an ex ante optimum. ${ }^{1}$ The other is that they take the terminal value of money to be exogenous, whereas we determine it within the model. In that respect, their model is a variant of standard static models of bilateral exchange with private information about valuations which are taken as fixed. The mechanism design problem in such settings is concerned with the extent to which trade can be achieved when it is mutually beneficial (see e.g. Myerson and Satterthwaite [12] and Kennan and Wilson [8]). In our model, on the other hand, the valuations depend on the mechanism, and much of the analytical effort in the paper is devoted to the implications of that dependence.

The benchmark to which we compare the role of lags is the case in which everyone is informed about the current realization when it occurs. Because money is indivisible and because it enters and disappears in a way which is a random version of lump-sum taxes and transfers, that benchmark does not display neutrality. For the range of parameters of interest, the benchmark has the following features: the price level and total output at a date depend only on the monetary realization for that date; the price level is increasing in the amount of money; the response of total output depends on rather fine details of the parameterization. In particular, while the fraction of meetings in which trade occurs is increasing in the amount of money because we assume that the amount of money is always small enough, the amount produced in each meeting is decreasing in the amount of money. We make no attempt to control which of those effects dominates in the benchmark model.

The existence of uninformed people is an obvious source of price level stickiness relative to the benchmark. When two uninformed people meet, their actions cannot depend on the current realization. In addition, in such meetings the per meeting output response to last period's realization, which is known, is less pronounced than is the response of informed people to the current realization. That happens because in both kinds of meeting, the participants are drawing inferences about the distribution of money in the next period and, by assumption, the current realization is more informative about that distribution than is last period's realization. In addition, as we will see, the truth-telling constraints in meetings between the informed and the uninformed are another source of stickiness. Thus, on several grounds, we expect to find that the price level is less responsive to monetary realizations the larger the set of uninformed people.

Total output relative to the benchmark behaves in a way that depends on what happens in meetings between the informed and the uninformed. An increase in the

\footnotetext{
${ }^{1}$ One benefit of describing an optimum is that it endogenizes how trade is conducted.
} 
amount of money increases the fraction of meetings between informed consumers and uninformed producers in which trade can occur. Moreover, because, as we show, there is trade in all those meetings and a level of production in each meeting which does not depend on the current state, such meetings are a source of larger total output effects relative to the benchmark. However, the meetings between informed producers and uninformed consumers provide a potential offset. If a monetary increase has sufficiently low probability, then the optimum has no-trade in meetings between informed producers and uninformed consumers when such an increase occurs. When that happens and if there are enough such meetings, then there can be negative impact effects on output. If the monetary increase occurs with high enough probability, then the optimum does not have no-trade and the offset does not occur. ${ }^{2}$ The optimum, by the way, does not have no-trade when a low probability monetary decrease occurs. Hence, impact effects can be asymmetric for increases and decreases.

The paper proceeds as follows. In section 2 , we describe the environment we study. In section 3, we describe the set of deterministic mechanisms we consider and the optimum problem. In section 4, we present our main results, a characterization of the optimum in what we take to be an interesting region of the parameter space: the region in which producer participation constraints are binding, but consumer participation constraints are not binding. In section 5, we present examples which display the effects described above. In section 6 , we discuss the role of randomization. In section 7 , we point out the differences between the optimal mechanism and bargaining in which consumers, whether informed or not, make take-it-or-leave-it offers. The paper ends with some concluding remarks, primarily about possible extensions of what we do. All the proofs appear in the Appendix.

\section{The environment}

We first set out the familiar background environment. Then we describe how money supply changes come about.

\subsection{The background environment}

Aside from the uncertainty about the stock of money, the environment is that in Wallace [15], which, in turn, is essentially that in Shi [13] and Trejos and Wright [14]. Time is discrete and the horizon is infinite. There are $N$ distinct, divisible, and perishable types of goods at each date and there is a $[0,1]$ continuum of each of $N$ specialization types of people, where $N \geq 3$. A specialization type- $n$ person consumes only good $n$ and produces only good $n+1$ (modulo $N$ ), for $n=1,2, \ldots, N$. Each person maximizes expected discounted utility with discount factor $\beta \in(0,1)$. Utility in a period is given by $u(x)-y$, where $x$ is the amount consumed and $y$ is the amount produced. The function $u$ is defined on $[0, \infty)$, is increasing, strictly concave, and satisfies $u(0)=0$ and $u^{\prime}(0)=\infty$.

In each period, people are randomly matched in pairs. Meetings are of two sorts: single-coincidence meetings, those between a type $n$ person (the producer) and a type

\footnotetext{
${ }^{2}$ Results somewhat like these are obtained by Jones and Manuelli.
} 
$n+1$ person (the consumer) for some $n$; and no-coincidence meetings, those in which neither person produces what the other consumes. (Because the number of types, $N$, exceeds two, there are no double-coincidence meetings.) We assume that people cannot commit to what they will do in future meetings.

Money consists of perfectly durable and indivisible objects which cannot be produced and which do not yield utility directly. We assume that each person can carry from one date to the next at most one unit of money. We also assume that each trader in a meeting is able to see the trading partner's specialization type, money holdings, and whether the person is informed or not, but is otherwise ignorant about the trading partner's history. The assumption that the set of informed people is common knowledge is a simplification. We will comment at the end on a version in which that is not the case.

\subsection{How changes in the amount of money come about}

The quantity of money follows an $S$-state Markov process. That is, there are $S$ potential levels for the stock of money: $m_{1}, m_{2}, \ldots, m_{S}$ where $m_{i} \in(0,1)$ and $m_{i}<$ $m_{i+1}$. Here $m_{i}$ is the state $i$ amount of money per specialization type. We let $\pi_{i j}$ denote the probability that the current state is $j$ given that the previous state is $i$ and let $\Pi$ denote the associated transition matrix. We assume that $\pi_{i j}>0$ which implies that $\Pi$ has a unique invariant distribution which assigns positive probability to each state.

We want changes in the money supply to come about in a way that gives no immediate information to the uninformed. That is accomplished by assuming that only informed people either gain or lose money and by assuming the following timing. At the end of each date after meetings have dissolved, the current amount of money is publicly announced. (Consequently, at that time, people differ only in money holdings, not in information.) Then, a randomly chosen subset of each specialization type, of measure $\lambda$, is selected and is informed about the new state. If the previous state is $i$, then the measure of newly informed with money is $m_{i} \lambda$ and the measure without money is $\left(1-m_{i}\right) \lambda$. If the new state is $j>i$, then a randomly chosen subset of the informed without money, a subset of measure $m_{j}-m_{i}$, is given a unit of money. If the new state is $j<i$, then a randomly chosen subset of the informed with money, a subset of measure $m_{i}-m_{j}$, loses a unit of money. Then meetings occur and the sequence is repeated.

In order to be able to have all changes in the amount of money be experienced by those who are informed, we need to assume that $\lambda$ is large enough relative to the monetary changes. The following assumptions accomplish that:

$$
m_{S} \leq \frac{1}{2} \text { and } \lambda \in\left[\frac{m_{S}-m_{1}}{m_{S}}, 1\right] .
$$

The following two-by-two table contains the fraction of each specialization type according to whether they are informed and whether they have money given that the previous state is $i$ and the current state is $j$. 
Table 1. Distribution when the previous state is $i$ and the current state is $j$.

\begin{tabular}{|c|c|c|c|}
\hline & 0 units of money & 1 unit of money & sums \\
\hline informed & $m_{i}-m_{j}+\left(1-m_{i}\right) \lambda \equiv \theta_{j i}$ & $m_{j}-(1-\lambda) m_{i} \equiv \lambda-\theta_{j i}$ & $\lambda$ \\
\hline uninformed & $(1-\lambda)\left(1-m_{i}\right)$ & $(1-\lambda) m_{i}$ & $1-\lambda$ \\
\hline sums & $1-m_{j}$ & $m_{j}$ & 1 \\
\hline
\end{tabular}

Lemma 1 confirms that (2.1) is sufficient to have all monetary changes impinge on the informed.

Lemma 1. If (2.1) holds, then $\theta_{j i}$ and $\lambda-\theta_{j i}$ are non-negative.

The description of the environment is now complete. As we will see, it turns out to be complicated because there are meetings in which there is asymmetric information. Such situations are unavoidable in the following sense. While it makes sense to study the extreme in which everyone is informed $(\lambda=1)$ and in which, therefore, there is no asymmetric information, it does not make sense to study the other extreme in which no one is informed $(\lambda=0)$. According to $(2.1)$, if no one is informed, then there cannot be monetary uncertainty. More generally, it is hard to think of formulations in which everyone is both symmetrically informed and less than fully informed about monetary realizations.

For any $\lambda$, the monetary uncertainty has obvious incentive effects in our model. It makes those without money, the potential producers, less willing to produce in order acquire money because (i) they may lose the money acquired before they get to spend it; and (ii) if they do not produce, then they may be given a unit. It also makes those with money, the potential consumers, more willing to spend money because (i) if they do not spend it, then they may lose it; and (ii) if they do spend it, then they may be given a unit. The magnitude of those effects depends on the monetary uncertainty; roughly speaking, it is larger the larger is the range of monetary variation, $m_{S}-m_{1}$, and the smaller is the persistence implied by $\Pi$.

\section{Mechanisms and the optimum problem}

In this section, we present our theory of what happens in the environment described above. We do this in three steps. The first step is to describe a set of Markov mechanisms. The only meetings in which anything can happen are called trade meetings, meetings which are single-coincidence meetings in which the potential consumer has a unit of money and the producer does not. What happens is assumed to depend on the previous realization and on announcements about the current realization. The second step is to describe the subset of mechanisms that we call incentive-feasible. For us, a mechanism is incentive-feasible if it satisfies two conditions: it is consistent with truth-telling and is consistent with ex post individual rationality in the sense that it gives expected non-negative gains from trade to each person as they leave the meeting. (This last restriction implies that production is zero except in trade meetings, which is why we can limit consideration to trade meetings.) In particular, an uninformed person in a meeting has a chance to say no to a trade after seeing the 
proposed trade, but the proposed trade does not in general reveal the current state to the uninformed person. ${ }^{3}$ The third step is our selection from the set of incentivefeasible mechanisms. Our selection is an ex ante optimum, one which maximizes the ex ante expected utility of a representative person.

\subsection{Markov mechanisms}

Let $\mathbb{S} \equiv\{0,1,2, \ldots, S\}$ be the set of individual information states. If $s$ denotes a generic element of $\mathbb{S}$, then $s=0$ means uninformed about the current state and $s>0$ means informed and that the current amount of money is $m_{s}$. We also let $\mathbb{S}_{+} \equiv\{1,2, \ldots, S\}$. A Markov mechanism is a pair of functions that describes output and the transfer of money in trade meetings. That is, we let $y\left(s^{c}, s^{p}, i\right): \mathbb{S} \times \mathbb{S} \times \mathbb{S}_{+} \rightarrow R_{+}$denote output in a trade meeting when the consumer announces information state $s^{c}$, the producer announces $s^{p}$, and the previous money-supply realization is $i$. We also let $a\left(s^{c}, s^{p}, i\right)$ : $\mathbb{S} \times \mathbb{S} \times \mathbb{S}_{+} \rightarrow\{0,1\}$ denote the money transfer (0 means no transfer, 1 means transfer) in such a meeting. We record this notion of a mechanism as a definition.

Definition 1. A mechanism is a pair $(y, a)$ where $y: \mathbb{S} \times \mathbb{S} \times \mathbb{S}_{+} \rightarrow R_{+}$and $a$ : $\mathbb{S} \times \mathbb{S} \times \mathbb{S}_{+} \rightarrow\{0,1\}$.

Notice that we are not permitting randomization. We discuss the potential role of randomization after we present our results for deterministic mechanisms.

\subsection{Incentive-feasible mechanisms}

One way to describe the class of mechanisms we study is as follows. At each meeting, there is a computer that is programmed at date 0 before people go off to their meetings, but is able to receive messages. At the beginning of a date, when the previous state is publicly announced, that information is received by each computer. In addition, the computer at a meeting is able to see who is informed and who is a producer and who is a consumer. If both are informed, then both simultaneously announce to the computer an element of $\mathbb{S}_{+}$. If one person is informed, then that person announces to the computer an element of $\mathbb{S}_{+}$. The uninformed person in the meeting does not see the announcement. In all cases, the computer then proposes a trade, which may be no trade. Then each person's choice is either to accept or reject, where rejection by either person implies no trade. Acceptance by both implies that the proposed trade is carried out. Whether trade occurs or not, the meeting ends. A mechanism is incentive feasible if it induces truth-telling and if the proposed trade satisfies individual rationality, and, therefore, induces acceptance.

Before we present the details, we can give an overview of the results. First, because we require non-negative expected gains from trade as people leave a meeting, there

\footnotetext{
${ }^{3}$ Note that the ex post stage here is taken to be the situation at the end of a meeting, rather the subsequent stage at which the current money supply is publicly announced (which is the point at which ex post welfare comparisons would conventionally be made). If it is assumed that all information available to the participants in each meeting is revealed in that meeting, then there are additional restrictions on the set of mechanisms. We are indebted to Nobuhiro Kiyotaki and Narayana Kocherlakota for conversations that helped steer us away from such a more restrictive specification.
} 
are never pure transfers between people. That is, we do not permit people to commit beforehand to the realization from a distribution; they have to be willing to carry out each realization. Therefore, production occurs if and only if money changes hands. And because the amount of money that changes hands is always one unit, in meetings between the informed and the uninformed the amount produced in exchange for money cannot depend on the current state. (If it did, then it would not be consistent with truth-telling by the informed person.) Therefore, in meetings between asymmetrically informed people, the mechanism has only to describe the amount produced and a partition of the set of current states into trade and no-trade sets. Moreover, those sets have to be ordered: if the producer is informed, then the set of states in which trade occurs has to be composed of those in which acquiring a unit of money is more valuable; while if the consumer is informed, then it has to be composed of those in which acquiring a unit of money is less valuable.

If there were no incentive constraints, then maximization of ex ante welfare would dictate that production always occur at a level at which the marginal utility of consuming equals the marginal disutility of producing - namely, at the solution to $u^{\prime}(y)=1$, which we denote $y^{*}$. For some regions of the parameter space $(\beta$ near enough to unity and $\Pi$ with sufficient persistence), an outcome with $y^{*}$ produced in every trade meeting is incentive-feasible. We view that to be an uninteresting outcome - in part because the price level would then be constant at $\frac{1}{y^{*}}$, not dependent on the monetary realization. Therefore, we focus on a region of the parameter space where output as high as $y^{*}$ is not incentive-feasible because it fails to satisfy producer individualrationality constraints. As we show below, that is easily accomplished-for example, by assuming that $\beta$ is sufficiently far from unity. ${ }^{4}$

With output below $y^{*}$, ex-ante welfare is increasing in output in every kind of meeting. Therefore, if producer individual-rationality constraints were the only constraints, then an optimum would satisfy those constraints with equality (that is, all of the gains from trade would accrue to the consumer). However, there are also consumer individual-rationality and truth-telling constraints. If the consumer and the producer have the same information about the future value of money, then when the level of output is such that the disutility of producing is just equal to the value of money, the utility of consuming this output must exceed the value of money. That is, if the producer's individual-rationality constraint binds in this situation, then the consumer's individual-rationality constraint is slack. We build on this result and, in effect, assume that the range of the support for the money supply, $m_{S}-m_{1}$, is small enough to make consumer individual-rationality constraints non-binding. In other words we assume that the informational discrepancies are never large enough to outweigh the surplus generated when the producer supplies enough output to make the producer's individual-rationality constraint bind. We show that, as a consequence of this assumption, it is optimal to have trade occur in every trade meeting except possibly in some of the meetings between informed producers and uninformed con-

\footnotetext{
${ }^{4}$ The possibility that output is constant at $y^{*}$ whenever trade occurs is an artifact of the unit upper bound on holdings. Output cannot be constant whenever trade occurs if individual money holdings are not restricted to the set $\{0,1\}$ and if individual discounted expected utility is strictly increasing in money holdings.
} 
sumers. Moreover, in all meetings, there are binding producer individual-rationality constraints. This is the partial characterization of the optimum that we give in section 4. It is partial because it holds only in a region of the parameter space and because it does not pin down the cut-off between trade and no-trade states in meetings between informed producers and uninformed consumers. Despite that, the characterization makes it very simple to find solutions for examples. It also allows us to prove that if $\Pi$ displays sufficient persistence, then it is optimal to shut down trade in meetings between informed producers and uninformed consumers whenever an increase in the amount of money occurs.

We now turn to details. In order to express the constraints on mechanisms implied by our specification of incentive-feasibility, it is helpful to have a notation for expected discounted utilities. We let $V_{k}(i)$ denote the expected utilities of someone who has $k$ units money just after the previous state $i$ is announced (and before the determination of the new state and the new set of informed people). We let $V_{k}$ denote the $S$-element vector with generic component $V_{k}(i)$. We also let $\Delta_{i} \equiv V_{1}(i)-V_{0}(i)$ and $\Delta \equiv V_{1}-V_{0}$.

It is also helpful to let $G_{k}\left(\iota^{c}, \iota^{p}, s, i\right)$ be the gain, relative to not trading, in a trade meeting of someone with $k$ units of money when the current realization is $s$ and the previous realization is $i$, and when both parties announce truthfully. Here, $\iota^{c} \in\{0,1\}$ indicates whether the consumer is informed $\left(\iota^{c}=1\right)$ or uninformed $\left(\iota^{c}=0\right)$, and $\iota^{p} \in\{0,1\}$ indicates, in the same way, whether the producer is informed. Then,

$$
\begin{aligned}
& G_{0}\left(\iota^{c}, \iota^{p}, s, i\right)=-y\left(\iota^{c} s, \iota^{p} s, i\right)+a\left(\iota^{c} s, \iota^{p} s, i\right) \beta \Delta_{s} \\
& G_{1}\left(\iota^{c}, \iota^{p}, s, i\right)=u\left(y\left(\iota^{c} s, \iota^{p} s, i\right)\right)-a\left(\iota^{c} s, \iota^{p} s, i\right) \beta \Delta_{s} .
\end{aligned}
$$

Notice that

$$
G_{0}\left(\iota^{c}, \iota^{p}, s, i\right)+G_{1}\left(\iota^{c}, \iota^{p}, s, i\right)=z\left(y\left(\iota^{c} s, \iota^{p} s, i\right)\right)
$$

where $z(y) \equiv u(y)-y$.

We can use these gain definitions to connect the discounted utilities to the mechanism. It is convenient to do this in two steps. We let $v_{k}(\iota, s, i):\{0,1\} \times \mathbb{S}_{+} \times \mathbb{S}_{+} \rightarrow R_{+}$ denote the expected discounted utility of someone who (i) holds $k \in\{0,1\}$ units of money, and (ii) is either informed $(\iota=1)$ or uninformed $(\iota=0)$ when the current state is $s$ and the previous state is $i$-all of this prior to meetings but after determination of the set of newly informed people and after additions or subtractions of money. These continuation values, which should only be thought of as a short-hand, satisfy

$$
\begin{aligned}
& N v_{0}(\iota, j, i)=N \beta V_{0}(j)+\left(\lambda-\theta_{j i}\right) G_{0}(1, \iota, j, i)+(1-\lambda) m_{i} G_{0}(0, \iota, j, i) \\
& N v_{1}(\iota, j, i)=N \beta V_{1}(j)+\theta_{j i} G_{1}(\iota, 1, j, i)+(1-\lambda)\left(1-m_{i}\right) G_{1}(\iota, 0, j, i) .
\end{aligned}
$$


In terms of them, the $V_{k}(i)$ are defined by

$$
\begin{aligned}
V_{0}(i)= & \sum_{j=1}^{S} \pi_{i j}\left[(1-\lambda) v_{0}(0, j, i)+\lambda v_{0}(1, j, i)\right]+ \\
& \sum_{j=i}^{S} \pi_{i j} \eta_{i j}\left(v_{1}(1, j, i)-v_{0}(1, j, i)\right) \\
V_{1}(i)= & \sum_{j=1}^{S} \pi_{i j}\left[(1-\lambda) v_{1}(0, j, i)+\lambda v_{1}(1, j, i)\right]- \\
& \sum_{j=1}^{i-1} \pi_{i j} \eta_{i j}\left(v_{1}(1, j, i)-v_{0}(1, j, i)\right),
\end{aligned}
$$

where

$$
\eta_{i j}=\left\{\begin{array}{c}
1-\frac{m_{j}}{m_{i}} \text { if } j<i \\
1-\frac{1-m_{j}}{1-m_{i}} \text { if } j \geq i
\end{array} .\right.
$$

It follows from $(2.1)$ that $\eta_{i j} \leq \lambda$.

Notice that equations $(3.4)$ with the $v_{k}(\iota, s, i)$ replaced by their expressions in (3.3) consist of $2 S$ equations in $V_{0}$ and $V_{1}$ that are linear for a given $(y, a)$. Moreover, with that substitution into equations (3.4), for a given $(y, a)$ the right-hand sides of equations (3.4) can be viewed as a function that maps $R^{2 S}$ to $R^{2 S}$. It is immediate that that mapping satisfies Blackwell's sufficient conditions for contraction: monotonicity and discounting. Therefore, equations (3.4) have a unique solution for a given $(y, a)$.

We next express the truth-telling and individual rationality constraints. For trade meetings with symmetric information, the individual-rationality constraints are as follows. For producers, they are

$$
G_{0}(1,1, s, i) \geq 0 \text { and } \sum_{j=1}^{S} \pi_{i j} G_{0}(0,0, j, i) \geq 0
$$

For consumers, they are

$$
G_{1}(1,1, s, i) \geq 0 \text { and } \sum_{j=1}^{S} \pi_{i j} G_{1}(0,0, j, i) \geq 0 .
$$

In each case, the first inequality pertains to an informed person who meets an informed person, while the second pertains to an uninformed person who meets an uninformed person. (Of course, for meetings between uninformed people, the condition is that there be expected gains from trade because the trade occurs before the participants know the current state.) When both are informed, truth-telling is always a Nash strategy (provided the mechanism proposes no trade when it receives mutually 
inconsistent reports). Hence, truth-telling in symmetrically informed meetings does not imply additional constraints.

We next consider trade meetings between the informed and the uninformed. If the informed is the producer, then the truth-telling constraint is

$$
a(0, s, i) \beta \Delta_{s}-y(0, s, i) \geq a\left(0, s^{\prime}, i\right) \beta \Delta_{s}-y\left(0, s^{\prime}, i\right),
$$

while if the informed is the consumer, then the truth-telling constraint is

$$
u(y(s, 0, i))-a(s, 0, i) \beta \Delta_{s} \geq u\left(y\left(s^{\prime}, 0, i\right)\right)-a\left(s^{\prime}, 0, i\right) \beta \Delta_{s} .
$$

Both must hold for all $\left(s, s^{\prime}\right) \in \mathbb{S}_{+} \times \mathbb{S}_{+}$.

Therefore, as noted above, if $a(0, s, i)=a\left(0, s^{\prime}, i\right)$, then the producer's truthtelling constraint implies $y(0, s, i)=y\left(0, s^{\prime}, i\right)$; while if $a(s, 0, i)=a\left(s^{\prime}, 0, i\right)$, then the consumer's truth-telling constraint implies $y(s, 0, i)=y\left(s^{\prime}, 0, i\right)$. Moreover, the individual-rationality constraints imply that there is positive production if and only if the consumer surrenders his or her unit of money. Therefore, we let

$$
S^{p}(i)=\left\{j \in \mathbb{S}_{+}: a(0, j, i)=1\right\} \text { and } S^{c}(i)=\left\{j \in \mathbb{S}_{+}: a(j, 0, i)=1\right\},
$$

and let $y(0, j, i)=Y^{p}(i)$ for $j \in S^{p}(i)$ and $y(j, 0, i)=Y^{c}(i)$ for $j \in S^{c}(i)$. Then the truth-telling constraints are equivalent to

$$
\max _{j \in \mathbb{S}_{+}-S^{p}(i)}\left\{\beta \Delta_{j}\right\} \leq Y^{p}(i) \leq \min _{j \in S^{p}(i)}\left\{\beta \Delta_{j}\right\}
$$

and

$$
\max _{j \in S^{c}(i)}\left\{\beta \Delta_{j}\right\} \leq u\left(Y^{c}(i)\right) \leq \min _{j \in \mathbb{S}_{+}-S^{c}(i)}\left\{\beta \Delta_{j}\right\}
$$

where we interpret the first to be vacuous if $S^{p}(i)$ or $\mathbb{S}_{+}-S^{p}(i)$ is empty and the second to be vacuous if $S^{c}(i)$ or $\mathbb{S}_{+}-S^{c}(i)$ is empty. These are the truth-telling constraints with regard to the partition of current states between the no-trade states and the trade states. They describe precisely the sense in which the gain from acquiring money has to be ordered across trade and no-trade states.

Finally, we have individual-rationality constraints for participants in these meetings. For an informed producer, the individual-rationality constraint is the second inequality in (3.11), while for an uninformed producer, it is

$$
Y^{c}(i) \leq \frac{1}{\gamma_{i}^{1}\left(S^{c}(i)\right)} \sum_{j \in S^{c}(i)} \pi_{i j}\left(\lambda-\theta_{j i}\right) \beta \Delta_{j},
$$

where

$$
\gamma_{i}^{1}\left(S^{c}(i)\right) \equiv \sum_{k \in S^{c}(i)} \pi_{i k}\left(\lambda-\theta_{k i}\right) .
$$

Here, $\pi_{i j}\left(\lambda-\theta_{j i}\right) / \gamma_{i}^{1}$ is the probability that the current state is $j$ given that $j \in S^{c}(i)$, that the previous state is $i$, and that the trading partner is an informed person with 
money. For an informed consumer, the individual-rationality constraint is the first inequality in (3.12), while for an uninformed consumer, it is

$$
\frac{1}{\gamma_{i}^{0}\left(S^{p}(i)\right)} \sum_{j \in S^{p}(i)} \pi_{i j} \theta_{j i} \beta \Delta_{j} \leq u\left(Y^{p}(i)\right),
$$

where

$$
\gamma_{i}^{0}\left(S^{p}(i)\right) \equiv \sum_{k \in S^{p}(i)} \pi_{i k} \theta_{k i} .
$$

Here, $\pi_{i k} \theta_{k i} / \gamma_{i}^{0}$ is the probability that the current state is $j$ given that $j \in S^{p}(i)$, that the previous state is $i$, and that the trading partner is an informed person without money. That is, the uninformed person in these meetings is calculating an expected gain from trade given the information implied by truth-telling, which reveals the set of trade states, and the knowledge about the current state implied by observing the money holding of someone who could have gained or lost money.

We can now record the definition of an incentive-feasible mechanism.

Definition 2. A mechanism $(y, a)$ is incentive feasible if there exist $V_{k}(i)$ that satisfy (3.1)-(3.14).

An immediate implication is that if $(y, a)$ is incentive feasible (in particular, satisfies the individual-rationality constraints), then $V_{0}(i)$ and $V_{1}(i)$ are non-negative.

\subsection{The planning problem}

We now describe our selection from the above set of incentive-feasible mechanisms. Our selection maximizes ex ante utility, denoted $Z$, which is defined as

$$
Z=\sum_{s=1}^{S} p_{s}\left[m_{s} V_{1}(s)+\left(1-m_{s}\right) V_{0}(s)\right] .
$$

Here $p_{i}$ denotes the invariant probability, implied by the $\pi_{i j}$, that the amount of money is $m_{i}$. This objective corresponds to starting up the economy by drawing the previous state from that invariant distribution and considering expected utility prior to such a draw and also prior to determining who starts with money.

The following lemma expresses $Z$ in terms of the parameters of the environment and the output levels in an arbitrary mechanism.

Lemma 2. For any mechanism,

$$
\begin{aligned}
Z= & \frac{(1-\lambda)^{2}}{(1-\beta) N} \sum_{i=1}^{S} p_{i} m_{i}\left(1-m_{i}\right) z[y(0,0, i)] \\
& +\frac{1}{(1-\beta) N} \sum_{i=1}^{S} p_{i} \sum_{s=1}^{S} \pi_{i s} \theta_{s i}\left(\lambda-\theta_{s i}\right) z[y(s, s, i)]
\end{aligned}
$$




$$
\begin{aligned}
& +\frac{1-\lambda}{(1-\beta) N} \sum_{i=1}^{S} p_{i} m_{i} \sum_{s=1}^{S} \pi_{i s} \theta_{s i} z[y(0, s, i)] \\
& +\frac{1-\lambda}{(1-\beta) N} \sum_{i=1}^{S} p_{i}\left(1-m_{i}\right) \sum_{s=1}^{S} \pi_{i s}\left(\lambda-\theta_{s i}\right) z[y(s, 0, i)] .
\end{aligned}
$$

Notice that output levels, the components of $y$, appear in $Z$ only by way of the function $z(z(x) \equiv u(x)-x)$ and that $a$, the money transfer variable, does not appear. Then, $z\left(y^{*}\right)$, where $u^{\prime}\left(y^{*}\right)=1$, is an upper bound on $z\left(\left(y\left(s, s^{\prime}, i\right)\right)\right.$. Because the weight on $z\left(\left(y\left(s, s^{\prime}, i\right)\right)\right.$ in $(3.16)$ is non-negative, we obtain an upper bound on $Z$ by having $y^{*}$ produced and consumed in all trade meetings. Because one-quarter of all singlecoincidence meetings is an upper bound on the number of trade meetings, it follows that an upper bound on $Z$ is $\frac{z\left(y^{*}\right)}{4 N(1-\beta)} \equiv Z^{*}$.

That bound on $Z$ implies a bound on the set of incentive-feasible outputs. Let $q=\min _{s}\left(p_{s} m_{s}\right)$. By $(3.15)$, if $(y, a)$ is incentive-feasible, then, by the non-negativity of the $V_{k}(s), V_{1}(s) \leq Z^{*} / q$ and $\Delta_{s} \leq Z^{*} / q$. By the individual-rationality constraints, it follows that each component of an incentive-feasible $y$ is bounded above by $\beta Z^{*} / q$. Moreover, because the $V_{k}(s)$ and, therefore, $\Delta_{s}$ are continuous in $y$, and because the constraints are expressed as weak inequalities, it follows that the optimum problem amounts to maximizing a continuous function over a non empty compact set. ${ }^{5}$ Therefore, a maximum exists.

\section{A Partial Characterization of the Optimum}

As noted above, we concentrate our study of optima in a region of the parameter space that is both interesting and tractable. To be interesting, the optimum should not be the unconstrained welfare maximum, $y\left(s, s^{\prime}, i\right) \equiv y^{*}$. Below (see lemma 5) we show that a simple sufficient condition implies that if $y$ is incentive feasible, then $y\left(s, s^{\prime}, i\right)<y^{*}$.

To state the condition, let $y_{\max }^{i}$ denote the unique positive solution for $x$ to

$$
x=\frac{\beta z(x)\left(1-m_{i}\right)}{N(1-\beta)} .
$$

If the distribution of money is degenerate at $m_{i}$, then $y_{\max }^{i}$ is the largest constant output that satisfies the producer's individual-rationality constraint (see [3], page 110). It follows from $u^{\prime}(0)=\infty$ that $y_{\max }^{i}>0$. Moreover, $y_{\max }^{i}$ is decreasing in $m_{i}$. We assume that

$$
y^{*} \geq y_{\max }^{1} .
$$

Because $y^{*}$ depends only on the function $z$ and because $y_{\max }^{1} \rightarrow 0$ as $\beta \rightarrow 0$ or $N \rightarrow \infty$, (4.2), which we maintain from now on, can always be satisfied. Under assumption

\footnotetext{
${ }^{5}$ Non emptiness is implied by the fact that no trade satisfies all the constraints. As will become evident later, the Theorem of the Maximum does not apply because the constraint set is not lower hemicontinuous.
} 
(4.2), we are assured that the objective $Z$ is increasing in each component of an incentive-feasible $y$, a fact which plays a role in our characterization.

In addition to keeping outputs below $y^{*}$-in fact, as we show, below $y_{\max }^{1}-$ we characterize the solution to the above optimum problem under one other assumption, which for now we leave implicit: we assume that any optimum satisfies $u\left(\beta \min _{s} \Delta_{s}\right) \geq y_{\max }^{1}$. Because, as we show below, (4.2) and incentive feasibility imply $\beta \max _{s} \Delta_{s} \leq y_{\max }^{1}$, this assumption implies that $u\left(\beta \min _{s} \Delta_{s}\right) \geq \beta \max _{s} \Delta_{s}$. That, in turn, implies that consumer individual-rationality constraints are not binding at an optimum. Later, we present a crude sufficient condition for satisfaction of $u\left(\beta \min _{s} \Delta_{s}\right) \geq y_{\max }^{1}$, one which holds for our examples.

Under the above assumptions, we describe necessary conditions for an optimum. The necessary conditions say that trade occurs in all trade meetings except possibly those between informed producers and uninformed consumers. Moreover, each positive production amount satisfies a producer individual-rationality constraint with equality. That fully characterizes the optimum except for the determination of the cut-off between current states in which trade does and does not occur in meetings between informed producers and uninformed consumers. We also show that for each specification of the sets $S^{p}(i)$, there is a unique monetary mechanism (one with some trade) that satisfies the necessary conditions. Then, because there is a finite number of possible specifications of the $S^{p}(i)$ sets, the optimum is easily found.

The result that trade always occurs when the consumer is informed and the producer is not uses the assumption that $u\left(\beta \min _{s} \Delta_{s}\right) \geq y_{\max }^{1}$. The intuition for the result is the following. Consider a mechanism in which $S^{c}(i) \equiv \mathbf{S}_{+}$. and in which production is given by (3.13) at equality. One might suppose that welfare could be improved if this is replaced by a mechanism with higher output in some states and no trade in others in that kind of meeting. For output to be higher, the right side of (3.13) has to be higher with $S^{c}(i) \neq \mathbf{S}_{+}$than with $S^{c}(i) \equiv \mathbf{S}_{+}$. But that, in turn, seems to require that $S^{c}(i)$ contain the high values of the components of $\Delta$ and that $\mathbf{S}_{+}-S^{c}(i)$ contain the low values, which would violate truth-telling for the informed consumer, (3.9). Hence, such an improvement seems not to be possible. Of course, this argument is far from complete, if only because it does not take into account that $\Delta$ depends on the $S^{c}(i)$ sets and the amounts produced.

\subsection{The value of acquiring money}

We begin by deriving some facts about $\Delta \equiv V_{1}-V_{0}$. We know that there is a unique $\Delta$ for each mechanism. We denote it $\Delta(y, a)$. The first result is about the weighted average of $\Delta$ that appears in the individual rationality constraint for uninformed producers in meetings with informed consumers, (3.13). It is part of the argument for the result that the optimum satisfies $S^{c}(i) \equiv \mathbf{S}_{+}$.

Lemma 3. If $(y, a)$ is incentive feasible, then $\Delta(y, a)$ satisfies,

$$
\frac{1}{\gamma_{i}^{1}\left(S^{c}(i)\right)} \sum_{j \in S^{c}(i)} \pi_{i j}\left(\lambda-\theta_{j i}\right) \beta \Delta_{j} \leq \frac{1}{\gamma_{i}^{1}\left(\mathbb{S}_{+}\right)} \sum_{j \in \mathbb{S}_{+}} \pi_{i j}\left(\lambda-\theta_{j i}\right) \beta \Delta_{j} .
$$


The proof makes use of truth-telling for informed consumers, the implied ordering of $\Delta$ between $S^{c}(i)$ and $\mathbb{S}_{+}-S^{c}(i)$.

The next lemma describes a mapping whose fixed point is $\Delta(y, a)$. In part, it eliminates, through substitution, the continuation values that were introduced as part of connecting the $V_{k}(i)$ to the allocation $(y, a)$.

Lemma 4. Let

$$
h(x ; y, a) \equiv b(y)+\beta C(a) x \text { for } x \in R^{S} .
$$

where the $S \times S$ matrix $C(a) \equiv\left[c_{i j}(a)\right]$ and the $S \times 1$ vector $b(y) \equiv\left[b_{i}(y)\right]$ are defined as

$$
\begin{gathered}
\frac{c_{i j}(a)}{\pi_{i j}}=(1-\lambda)\left[1-\frac{1-\lambda}{N} a(0,0, i)-\frac{\theta_{j i}}{N} a(0, j, i)-\frac{\lambda-\theta_{j i}}{N} a(j, 0, i)\right]+ \\
\left(\lambda-\eta_{i j}\right)\left\{1-\frac{\lambda}{N} a(j, j, i)-\frac{(1-\lambda)}{N}\left[\left(1-m_{i}\right) a(j, 0, i)+m_{i} a(0, j, i)\right]\right\}
\end{gathered}
$$

and

$$
\begin{aligned}
N b_{i}(y)= & (1-\lambda)^{2}\left[\left(1-m_{i}\right) u(y(0,0, i))+m_{i} y(0,0, i)\right]+ \\
& (1-\lambda) \sum_{j=1}^{S} \pi_{i j}\left[\theta_{j i} u(y(0, j, i))+\left(\lambda-\theta_{j i}\right) y(j, 0, i)\right]+ \\
& \sum_{j=1}^{S} \pi_{i j}\left(\lambda-\eta_{i j}\right)\left[\theta_{j i} u(y(j, j, i))+\left(\lambda-\theta_{j i}\right) y(j, j, i)\right]+ \\
& (1-\lambda) \sum_{j=1}^{S} \pi_{i j}\left(\lambda-\eta_{i j}\right)\left[\left(1-m_{i}\right) u(y(j, 0, i))+m_{i} y(0, j, i)\right] .
\end{aligned}
$$

Then, (i) $\Delta(y, a)$ is a fixed point of $h(\cdot ; y, a)$; (ii) $h(\cdot ; y, a)$ satisfies Blackwell's sufficient conditions for contraction; (iii) if $h\left(\Delta(y, a) ; y^{\prime}, a^{\prime}\right) \geq \Delta(y, a)$, then $\Delta\left(y^{\prime}, a^{\prime}\right) \geq \Delta(y, a)$; and (iv) if $y^{\prime} \geq y$, then $\Delta\left(y^{\prime}, a\right) \geq \Delta(y, a)$.

The next lemma, which uses assumption (4.2), shows that satisfaction of producer individual-rationality constraints implies that $y_{\max }^{1}$ is an upper bound on $\beta \Delta_{i}(y, a)$ and on output.

Lemma 5. If $(y, a)$ satisfies producer individual-rationality constraints and has constant output over trade states in meetings between informed and uninformed, then $y\left(s, s^{\prime}, i\right) \leq \beta \max _{i} \Delta_{i}(y, a) \leq y_{\max }^{1}$.

We are now ready for the characterization.

\subsection{The characterization}

Proposition 1 gives necessary conditions for an optimum.

Proposition 1. If $(y, a)$ is optimal and satisfies $u\left(\beta \min _{s} \Delta_{s}(y, a)\right) \geq y_{\max }^{1}$, then (i) $S^{c}(i) \equiv \mathbb{S}_{+}$, 


$$
\begin{gathered}
Y^{c}(i)=\frac{1}{\gamma_{i}^{1}\left(\mathbb{S}_{+}\right)} \sum_{j \in \mathbb{S}_{+}} \pi_{i j}\left(\lambda-\theta_{j i}\right) \beta \Delta_{j}(y, a) \text { for all } i \in \mathbb{S}_{+}, \\
a(0,0, i)=1 \text { and } y(0,0, i)=\sum_{j=1}^{S} \pi_{i j} \beta \Delta_{j}(y, a) \text { for all } i \in \mathbb{S}_{+}, \\
a(j, j, i)=1 \text { and } y(j, j, i)=\beta \Delta_{j}(y, a) \text { for all }(i, j) \in \mathbb{S}_{+} \times \mathbb{S}_{+},
\end{gathered}
$$

and

$$
Y^{p}(i)=\min _{j \in S^{p}(i)}\left\{\beta \Delta_{j}(y, a)\right\} \text { for all } i \in \mathbb{S}_{+} ;
$$

(ii) for each $i, S^{p}(i)$ is not empty; and (iii) if $\Delta_{j}(y, a)=\Delta_{k}(y, a)$, then for each $i$, either $j, k \in S^{p}(i)$ or $j, k \in \mathbb{S}_{+}-S^{p}(i)$.

The rather lengthy proof proceeds by contradiction. We first suppose that $(y, a)$ is optimal, but does not satisfy (i). Then we consider $\left(y^{\prime}, a^{\prime}\right)$ given by (4.7)-(4.10), but with $\Delta(y, a)$ inserted on the right-hand sides of (4.7)-(4.10). Then incentivefeasibility of $y$ implies that $y^{\prime} \geq y$. The main part of the proof involves showing that $h_{i}\left(\Delta(y, a) ; y^{\prime}, a^{\prime}\right) \geq \Delta_{i}(y, a)=h_{i}(\Delta(y, a) ; y, a)$, where $h_{i}$ is the $i$-th component of the mapping defined in (4.4). Given that inequality, the third part of lemma 4 implies that $\Delta\left(y^{\prime}, a^{\prime}\right) \geq \Delta(y, a)$. It follows that $\left(y^{\prime}, a^{\prime}\right)$ satisfies all the producer individual-rationality constraints. Moreover, by lemma $5, \beta \Delta_{j}\left(y^{\prime}, a^{\prime}\right) \leq y_{\max }^{1}$. Therefore, $u\left(\beta \min _{s} \Delta_{s}\left(y^{\prime}, a^{\prime}\right)\right) \geq u\left(\beta \min _{s} \Delta_{s}(y, a)\right) \geq y_{\max }^{1} \geq \beta \max _{s} \Delta_{s}\left(y^{\prime}, a^{\prime}\right)$. It follows that $\left(y^{\prime}, a^{\prime}\right)$ satisfies all the consumer individual-rationality constraints. It also satisfies truth-telling for the informed consumer because it has trade in every state when the consumer is informed. But $\left(y^{\prime}, a^{\prime}\right)$ may or may not satisfy truth-telling for informed producers. If it does, then we have a contradiction because $y_{\max }^{1} \geq y^{\prime} \geq y$ and we have either increased output or replaced trade by no trade, both of which increase $Z$. If not, then there is some previous state $i$ and some current state $r$ such that $\beta \Delta_{r}\left(y^{\prime}, a^{\prime}\right)>Y^{p}(i)^{\prime} \geq \beta \Delta_{r}(y, a)$. We then consider $\left(y^{\prime}, a^{\prime \prime}\right)$, where $a^{\prime \prime}$ differs from $a^{\prime}$ only in adding state $r$ to $S^{p}(i)$. We can show that $\Delta\left(y^{\prime}, a^{\prime \prime}\right) \geq \Delta(y, a)$. Repetition of this argument at most $S$ times leads to trade in every state, which renders truth-telling vacuous. Parts (ii) and (iii) are proved using simple versions of the same argument.

The next lemma shows that for a given specification of the $S^{p}(i)$ sets there is a unique monetary mechanism that satisfies condition (i) of proposition 1.

Lemma 6. Given $S^{p}(i)$ for each $i \in \mathbb{S}_{+}$, there exists a unique monetary mechanism that satisfies (4.7)-(4.10).

The proof shows that a mapping whose fixed points coincide with solutions to (4.7)(4.10) and (9.13) has a unique positive fixed point. The proof relies on monotonicity and concavity of the mapping (see [7]). One by-product of the proof is the result that the solution to (4.7)-(4.10) and (9.13) can be obtained by a simple iterative scheme that does not require solving simultaneous equations.

Proposition 1 and lemma 6 give us a simple procedure for finding the optimum for a given environment. For each specification of the $S^{p}(i)$ sets that are not empty, 
obtain the unique monetary mechanism that satisfies (4.7 )-(4.10). Discard those that are inconsistent with truth-telling for the informed producer. Among those that remain, the optimum is the one that gives the highest $Z$. Because $S^{p}(i) \equiv \mathbb{S}_{+}$satisfies truth-telling, there is at least one monetary mechanism that is incentive-feasible.

We now show that the maximized objective is continuous in $\Pi$ provided that $\pi_{i i}$ is bounded away from 0 for each $i$. We establish such continuity in two steps. First, we show that for given $S^{p}(i)$ sets, the unique monetary mechanism that satisfies (4.7)-(4.10) is continuous in $\Pi$.

Lemma 7. Let $S^{p}(i)$ for $i \in \mathbb{S}_{+}$be given. Let $\mathbb{P}=\left\{\Pi: \pi_{i i} \geq \alpha>0\right.$ for all $\left.i\right\}$. Then the unique monetary mechanism that satisfies (4.7)-(4.10) is continuous in $\Pi$ for $\Pi \in \mathbb{P}$.

Now we give the main continuity result.

Proposition 2. If the optimum satisfies $u\left(\beta \min _{s} \Delta_{s}\right) \geq y_{\max }^{1}$, then the maximized objective is continuous in $\Pi$ for $\Pi$ such that $\pi_{i j}$ is positive and $\pi_{i i}$ is bounded away from 0 .

While the proof uses lemma 7, it must do more than appeal to the fact that the maximum of continuous functions is continuous. The unique monetary mechanism that satisfies (4.7)-(4.10) for given $S^{p}(i)$ sets may satisfy truth-telling for informed producers for some $\Pi^{\prime} s$ and not others. We can show that if $\Pi_{0}$ is a critical point in terms of such truth-telling (truth-telling holds at $\Pi_{0}$ but not in the neighborhood of $\Pi_{0}$ ), then necessary condition (iii) in proposition 1 fails at $\Pi_{0}$. That, in turn, implies that the maximum cannot be at such a critical point. That and lemma 7 imply that the maximized objective is continuous.

There is a sense in which optima are not very interesting if they always satisfy $S^{p}(i) \equiv \mathbb{S}_{+}$. To see why that does not always happen, we begin with an informal argument for a simple case. Consider $S=2$ and a symmetric transition matrix,

$$
\Pi=\left[\begin{array}{cc}
1-\varepsilon & \varepsilon \\
\varepsilon & 1-\varepsilon
\end{array}\right] .
$$

When $\varepsilon=0$, the model is one with a constant money supply. Then the optimum is $y\left(\iota^{c} i, \iota^{p} i, i\right)=y_{\max }^{i}($ see $(4.1))$. Now suppose that $\varepsilon>0$, but small. In this model, the current amount of money influences the producer's individual-rationality constraint because it influences the distribution of the amount of money next period. Thus, if $\varepsilon$ is near 0 , then we expect that the producer's individual-rationality constraint is slacker the smaller is the current money supply. Now consider the meetings between informed producers and uninformed consumers. If last period's state is $m_{1}$ and if production is positive in both current states, then output has to be independent of the current state. If, instead, there is no-trade when the current state is $m_{2}$, then output can be higher when the current state is $m_{1}$. If $\varepsilon$ is sufficiently close to 0 , then it should be optimal to have no-trade when the current state is $m_{2}$. Matters are different if last period's state is $m_{2}$. Then in order to get higher output in some state than output that is constant across current states, there would have to be no-trade 
when the current state is $m_{2}$. But that would not be beneficial because the conditional probability of $m_{2}$ is high.

We formalize that discussion as follows.

Proposition 3. Let $\left\{\Pi_{k}\right\} \rightarrow I$ (the identity matrix) and be such that the corresponding sequence of stationary probability vectors $\left\{p_{k}\right\}$ converges to a strictly positive vector. Assume that the optimum for any $\Pi$ in the neighborhood of $\Pi=I$ satisfies $u\left(\beta \min _{s} \Delta_{s}\right) \geq y_{\max }^{1}$. There exists $K$ such that if $k>K$, then the optimum for $\Pi_{k}$ has no trade between informed producers and uninformed consumers whenever the current state, $j$, exceeds the previous state, $i$.

The main idea for the proof is similar to the discussion given above for $S=2$.

Now that we have characterized the optimum, a comment about implementing it is in order. The optimum we have described is easy to implement, and without using the fiction of a computer at each meeting that receives reports and is updated when the previous state is publicly announced. At each trade meeting and conditional on the knowledge that is common to the two people in the meeting, the optimum has a single positive trade proposal. Indeed, this is a feature of any incentive-feasible mechanism. Therefore, we can let the two people play a simultaneous move game in which the strategies are simply \{trade, no-trade\}. If both say trade, then the positive trade proposal is carried out. If either says no-trade, then there is no trade and each goes on to the next date. In part because the optimum has positive trade in every state when the consumer is informed and the producer is not, it is a dominant strategy for the consumer in every trade meeting to play trade. Given that play by the consumer, it is a best response for the producer to play the strategy that implements the optimum.

\subsection{Sufficient conditions for $u\left(\beta \min _{s} \Delta_{s}\right) \geq y_{\max }^{1}$}

Our last task in this section is to provide a sufficient condition for assuring that any optimum satisfies $u\left(\beta \min _{s} \Delta_{s}\right) \geq y_{\max }^{1}$, the hypothesis of propositions 1-3. The sufficient condition uses a lower bound on the optimal magnitude of $Z$, a bound we call $Z_{\min }$. As part of the proof that shows that a small enough range for the support of the money supply is sufficient to insure $u\left(\beta \min _{s} \Delta_{s}\right) \geq y_{\max }^{1}$ (lemma 9 ), we provide one such $Z_{\min }$. More generally, $Z_{\min }$ could be obtained from any lemma 6 mechanism that is incentive feasible.

Lemma 8. For each $k \in \mathbb{S}_{+}$, let $\Delta^{k} \in R^{S}$ be defined by $\Delta_{j}^{k}=y_{\max }^{1} / \beta$ for $j \neq k$ and $\beta \Delta_{k}^{k}=u^{-1}\left(y_{\max }^{1}\right)$. Then let $y^{k}$ be defined by

$$
\begin{aligned}
y^{k}(j, 0, i) & =\frac{1}{\gamma_{i}^{1}\left(\mathbb{S}_{+}\right)} \sum_{s=1}^{S} \pi_{i s}\left(\lambda-\theta_{s i}\right) \beta \Delta_{s}^{k} \\
y^{k}(j, j, i) & =y^{k}(0, j, i)=\beta \Delta_{j}^{k}, y^{k}(0,0, i)=\beta \sum_{s=1}^{S} \pi_{i s} \Delta_{s}^{k},
\end{aligned}
$$


for all $i, j \in \mathbb{S}_{+}$. Also, let $Z\left(y^{k}\right)$ be ex ante welfare implied by $y^{k}$ evaluated from (3.16). Let $Z_{\min }$ be the ex ante welfare implied by some incentive-feasible mechanism. If there exists $Z_{\min }$ such that $Z\left(y^{k}\right) \leq Z_{\min }$ for each $k \in \mathbb{S}_{+}$, then any optimum satisfies $u\left(\beta \min _{s} \Delta_{s}\right) \geq y_{\max }^{1}$.

The proof in outline is as follows. If the optimum violates $u\left(\beta \min _{s} \Delta_{s}\right) \geq y_{\max }^{1}$, then for some $k \in \mathbb{S}_{+}, \beta \Delta_{k}<u^{-1}\left(y_{\max }^{1}\right)$. (Notice that $u^{-1}\left(y_{\max }^{1}\right)<y_{\max }^{1}$.) If so, then, as we show, $y^{k}$ is strictly greater than any optimal $y$, and, hence, $Z\left(y^{k}\right)$ is strictly greater than the optimum $Z$. Therefore, if $Z\left(y^{k}\right) \leq Z_{\min }$ for each $k \in \mathbb{S}_{+}$, then we have a contradiction and the optimum cannot satisfy $u\left(\beta \min _{s} \Delta_{s}\right)<y_{\max }^{1}$.

Lemma 8 is a crude result because the specification in (4.12) is a crude upper bound on an incentive feasible $y$ given that $\beta \Delta_{k}<u^{-1}\left(y_{\max }^{1}\right)$ for some $k$. Despite that crudeness, the next result says that the hypothesis of lemma 8 can always be met if the range for the support of the money supply is sufficiently small.

Lemma 9. There exists $\varepsilon>0$ such that if $m_{S}-m_{1}<\varepsilon$, then the hypothesis of lemma 8 holds.

In the proof of this lemma, we construct a $Z_{\min }$ from a mechanism with constant output and trade in every trade meeting. Then we show that as $m_{S}-m_{1} \rightarrow 0$, this constant output approaches $y_{\max }^{1}$. That, in turn, guarantees that the implied $Z_{\min }$ satisfies the hypothesis of lemma 8.

To summarize, the following two-step algorithm permits us to determine whether a given environment satisfies conditions that are sufficient to insure that any optimum satisfies $y_{\max }^{1} \leq y^{*}$ and $u\left(\beta \min \Delta_{i}\right) \geq y_{\max }^{1}$.

(i). Compute $y_{\max }^{1}$ and $y^{*}$ and confirm that $y_{\max }^{1} \leq y^{*}$. (Then lemma 5 implies that any optimum satisfies $y_{\max }^{1} \geq \beta \max \Delta_{i}$.)

(ii). Attempt to find some $Z_{\min }$ (the optimum from lemma 6 and the construction in the proof of lemma 9 are possibilities) for which the hypothesis of lemma 8 holds.

\section{Examples}

We present three examples designed to emphasize the consequences for aggregates of whether trade is shut down in some meetings between informed producers and uninformed consumers. As discussed in the introduction, we expect any such shutting

down to affect the magnitude of the total output response to changes in the amount of money relative to what happens in the benchmark case of everyone informed $(\lambda=1)$.

The aggregates we study are total output and price level. For each example, we present the optimal Markov process for total output and the price level. Let $Y_{i j}$ and $P_{i j}$ be total output and the price level, respectively, when the previous state is $i$ and the current state is $j$. For a given mechanism, total output is the appropriate sum of outputs over meetings:

$$
\begin{aligned}
N Y_{i j}= & m_{i}\left(1-m_{i}\right)(1-\lambda)^{2} y(0,0, i)+\left(\lambda-\theta_{j i}\right) \theta_{j i} y(j, j, i)+ \\
& \left(\lambda-\theta_{j i}\right)\left(1-m_{i}\right)(1-\lambda) y(j, 0, i)+m_{i} \theta_{j i}(1-\lambda) y(0, j, i) .
\end{aligned}
$$


We take $P_{i j}$ to be total nominal output, denoted $X_{i j}$, divided by $Y_{i j}$, the usual definition of the total output deflator. ${ }^{6}$ Because one unit of money is traded in every meeting in which trade occurs, for a given mechanism total nominal output is the appropriate sum of the number of trades. That is,

$$
\begin{aligned}
N X_{i j}= & m_{i}\left(1-m_{i}\right)(1-\lambda)^{2} a(0,0, i)+\left(\lambda-\theta_{j i}\right) \theta_{j i} a(j, j, i)+ \\
& \left(\lambda-\theta_{j i}\right)\left(1-m_{i}\right)(1-\lambda) a(j, 0, i)+m_{i} \theta_{j i}(1-\lambda) a(0, j, i) .
\end{aligned}
$$

Notice that the right-hand side of (5.2) is the right-hand side of (5.1) with $y\left(s, s^{\prime}, i\right)$ replaced by $a\left(s, s^{\prime}, i\right)$. The pair $\left(Y_{i j}, P_{i j}\right)$ occurs with probability $p_{i} \pi_{i j}$, where, as above, $p_{i}$ denotes the stationary probability that the state is $i$. Therefore, the pair $\left(Y_{i j}, P_{i j}\right)$ and the exogenous probability $p_{i} \pi_{i j}$ for each $(i, j)$ completely describe the Markov process for total output and the price level. For each example, we report the pair $\left(Y_{i j}, P_{i j}\right)$ implied by (5.1) and (5.2) for the optimal mechanism.

The first two examples share all but one feature. Both have $S=2$ and the symmetric $\Pi$ given in (4.11). We let $u$ be the square-root function-mainly because it is a simple function that satisfies our general assumptions. For that choice of $u$, $y^{*}=\frac{1}{4}$. We also let $N=3$, the minimum consistent with no double-coincidence meetings. For both examples, the support for the money supply is $\left(m_{1}, m_{2}\right)=\left(\frac{4}{9}, \frac{1}{2}\right)$. The quantity $\frac{1}{2}$ is the amount of money that maximizes the probability of trade meetings for an economy with a constant money supply. If $m_{2}=\frac{1}{2}$, then $m_{1}=\frac{4}{9}$ implies that we are studying roughly $10 \%$ increases and decreases in the amount of money in these examples. We choose $\beta$ so that $y_{\max }^{1}=y^{*}$, which gives $\beta=\frac{27}{32}$. That, in turn, implies that $y_{\max }^{2}=\frac{81}{361}=.224$. The two examples differ regarding $\varepsilon$. As noted above, for $S=2$ and for a symmetric $\Pi$, we expect to have trade occur all the time if $\varepsilon$ in (4.11) is not too close to zero and we expect to have no-trade occur if $\varepsilon$ is small enough when $(i, j)=(1,2)$. For each $\varepsilon$, we report results for three values of $\lambda$ : $\lambda=\frac{1}{9}$, the minimum consistent with $(2.1) ; \lambda=\frac{11}{20}$, the magnitude which maximizes the probability of meetings between informed producers and uninformed consumers; and $\lambda=1$, the benchmark.

We choose magnitudes of $\varepsilon$ to display the two possibilities for shutting down trade and so that the resulting specifications satisfy the sufficient condition of section 4.3. Example 1 has $\varepsilon=0.1$, which implies that trade always occurs. Example 2 has $\varepsilon=.0025$, which turns out to be small enough to get no-trade in meetings between informed producers and uninformed consumers when $(i, j)=(1,2) \cdot{ }^{7}$ The following two tables contain the results, with total output scaled up by $10^{5}$.

\footnotetext{
${ }^{6}$ We must use a price index because, in general, the price of output is dependent on the kind of meeting. The price in a meeting is $1 / y\left(s, s^{\prime}, i\right)$ for $\mathrm{s}$ and $s^{\prime} \in \mathbb{S}$. Therefore, for given past and current states, the price varies with who in the meeting is informed. Therefore, an outside observer collecting such prices would have to use an index to compute $a$ price level.

${ }^{7}$ The section 4.3 sufficient condition is satisfied for examples 1 and 2 , because the value of $Z\left(y^{k}\right)$ (which is the same for each $k$, by the symmetry of the example) never rises above .12 , and this is below the values of $Z$ shown in the tables. Note that $\Delta_{1}>\Delta_{2}$ so that any mechanism that sets $a(0,2,1)=0$ satisfies the producer truth-telling constraint. Also, the values of $\Delta_{1}$ and $\Delta_{2}$ imply that $u\left(\beta \Delta_{2}\right)>\beta \Delta_{2}$, so the consumer individual rationality constraints are not binding. Therefore, the value of $Z$ we report is a valid choice for $Z_{\min }$.
} 
Table 2. Example 1: $\Pi$ given by (4.11) with $\varepsilon=\frac{1}{10}$

\begin{tabular}{|c|c|c|c|c|c|c|c|}
\hline$\lambda$ & $Z$ & $j$ & $Y_{1 j}$ & $Y_{2 j}$ & $P_{1 j}$ & $P_{2 j}$ & $\Delta$ \\
\hline \multirow{3}{*}{1} & \multirow{3}{*}{132033} & 1 & 1869 & 1869 & 4.405 & 4.405 & .2691 \\
\hline & & 2 & 1800 & 1800 & 4.630 & 4.630 & .2560 \\
\hline & & $\frac{X_{i i^{\prime}}-X_{i i}}{X_{i j}}$ & $-3.67 \%$ & $3.81 \%$ & $5.11 \%$ & $-4.86 \%$ & $-4.86 \%$ \\
\hline \multirow{3}{*}{$\frac{11}{20}$} & \multirow{3}{*}{131924} & 1 & 1821 & 1792 & 4.521 & 4.592 & .2654 \\
\hline & & 2 & 1823 & 1792 & 4.570 & 4.649 & .2545 \\
\hline & & $\frac{X_{i i^{\prime}}-X_{i i}}{X_{i i}}$ & $0.15 \%$ & $-0.00 \%$ & $1.08 \%$ & $-1.23 \%$ & $-4.13 \%$ \\
\hline \multirow{3}{*}{$\frac{1}{9}$} & \multirow{3}{*}{131988} & 1 & 1840 & 1780 & 4.472 & 4.625 & .2674 \\
\hline & & 2 & 1869 & 1802 & 4.459 & 4.625 & .2553 \\
\hline & & $\frac{X_{i i^{\prime}}-X_{i i}}{X_{i i}}$ & $1.56 \%$ & $-1.24 \%$ & $-0.29 \%$ & $-0.00 \%$ & $-4.52 \%$ \\
\hline
\end{tabular}

Table 3. Example 2: $\Pi$ given by (4.11) with $\varepsilon=\frac{1}{400}$

\begin{tabular}{|c|c|c|c|c|c|c|c|}
\hline$\lambda$ & $Z$ & $j$ & $Y_{1 j}$ & $Y_{2 j}$ & $P_{1 j}$ & $P_{2 j}$ & $\Delta$ \\
\hline \multirow{3}{*}{1} & \multirow{3}{*}{.132325} & 1 & 2051 & 2051 & 4.012 & 4.012 & .29540 \\
\hline & & 2 & 1869 & 1869 & 4.458 & 4.458 & .26587 \\
\hline & & $\frac{X_{i i^{\prime}}-X_{i i}}{X_{i i}}$ & $-8.87 \%$ & $9.73 \%$ & $11.12 \%$ & $-9.99 \%$ & $-9.99 \%$ \\
\hline \multirow{3}{*}{$\frac{11}{20}$} & \multirow{3}{*}{132291} & $\frac{\Lambda_{i i}}{1}$ & 2050 & 1906 & 4.015 & 4.318 & .29524 \\
\hline & & 2 & 1598 & 1869 & 4.171 & 4.458 & .26584 \\
\hline & & $\frac{X_{i i^{\prime}}-X_{i i}}{X_{i}}$ & $-22.03 \%$ & $1.97 \%$ & $3.89 \%$ & $-3.14 \%$ & $-9.96 \%$ \\
\hline \multirow{3}{*}{$\frac{1}{9}$} & \multirow{3}{*}{.132324} & 1 & 2051 & 1847 & 4.013 & 4.457 & .29538 \\
\hline & & 2 & 2055 & 1870 & 4.015 & 4.457 & .26586 \\
\hline & & $\frac{X_{i i^{\prime}}-X_{i i}}{X_{i i}}$ & $0.23 \%$ & $-1.23 \%$ & $0.05 \%$ & $0.00 \%$ & $-9.99 \%$ \\
\hline
\end{tabular}

In the tables, the rows labeled $\frac{X_{i i^{\prime}}-X_{i i}}{X_{i i}}$ contain percentage changes of switching states relative to not switching states, except when they pertain to $\Delta$. For $\Delta$, the entry is $\frac{\Delta_{2}-\Delta_{1}}{\Delta_{1}}$. In addition to impact effects, the outcomes associated with any pattern of realizations for the money supply can be identified. For example, if $\varepsilon=0.1$ and $\lambda=\frac{11}{20}$ and if the sequence of money realizations starting at date $t-1$ is $\left(m_{1}, m_{1}, m_{2}, m_{2}, m_{1}\right)$, then the sequence for the price level starting at date $t$ is $(4.521,4.570,4.649,4.592)$.

As we suggested in the introduction, impact effects depend on whether trade is ever shut down and on the probability of meetings between informed producers and uninformed consumers. For $\varepsilon=0.1$, when there is always trade, there are positive impact effects on output relative to the benchmark and small effects on the price level. For $\varepsilon=0.0025$, when there is no-trade when informed producers meet uninformed consumers and the money supply has increased, the price level again displays little initial movement relative to what happens in the benchmark. In fact, when $\lambda=\frac{1}{9}$, the price moves hardly at all initially. The impact effects on output also depend on $\lambda$. When $\lambda=\frac{11}{20}$, which maximizes the probability of meetings between informed producers and uninformed consumers, an increase in the amount of money produces a large decline relative to what happens in the benchmark. When $\lambda=\frac{1}{9}$, such meetings are rare, because most informed people have money when the amount of money increases. In this case, there is a large positive impact effect on output relative 
to the benchmark. As might be expected, the highest level of ex ante welfare, $Z$, is achieved when everyone is informed. However, ex ante welfare is not monotone in $\lambda .^{8}$

Example 3 is designed to have non monotone impact effects of increases in the amount of money on output. The example has $S=3$ and combines features of examples 1 and 2 so that large increases in the amount of money produce a shutting down of trade, but small increases do not. We continue to let $u$ be the squareroot function, and let $N=3$. The support for the money supply is $\left(m_{1}, m_{2}, m_{3}\right)=$ $\left(\frac{5}{12}, \frac{4}{9}, \frac{1}{2}\right) \approx(.4167, .4444, .5000)$, while $\beta=\frac{36}{43}$ so that, again, $y_{\max }^{1}=y^{*}$. For $\Pi$, we chose,

$$
\Pi=\left[\begin{array}{ccc}
1-\varepsilon_{1}-\varepsilon_{2} & \varepsilon_{1} & \varepsilon_{2} \\
\varepsilon_{1} & 1-2 \varepsilon_{1} & \varepsilon_{1} \\
\varepsilon_{2} & \varepsilon_{1} & 1-\varepsilon_{1}-\varepsilon_{2}
\end{array}\right],
$$

with $\left(\varepsilon_{1}, \varepsilon_{2}\right)=(.0500, .0025)$. Even though the range for the money supply is larger for this example, it remains true that the hypothesis of lemma 8 holds. ${ }^{9}$ For this example, we report only $\lambda=1$ and $\lambda=\frac{11}{20}$. The results are in Table 4 .

Table 4. Example 3: $\Pi$ in (5.3) with $\left(\varepsilon_{1}, \varepsilon_{2}\right)=\left(\frac{1}{20}, \frac{1}{400}\right)$

\begin{tabular}{|c|c|c|c|c|c|c|c|c|c|}
\hline$\lambda$ & $Z$ & $j$ & $Y_{1 j}$ & $Y_{2 j}$ & $Y_{3 j}$ & $P_{1 j}$ & $P_{2 j}$ & $P_{3 j}$ & $\Delta$ \\
\hline \multirow{3}{*}{1} & \multirow{3}{*}{1259} & 1 & 1953 & 1953 & 1953 & 4.148 & 4.148 & 4.148 & .2879 \\
\hline & & 2 & 1858 & 1858 & 1858 & 4.430 & 4.430 & 4.430 & .2696 \\
\hline & & 3 & 1751 & 1751 & 1751 & 4.759 & 4.759 & 4.759 & .2510 \\
\hline \multirow{3}{*}{$\frac{11}{20}$} & \multirow{3}{*}{.1257} & 1 & 1874 & 1797 & 1755 & 4.324 & 4.508 & 4.617 & .2810 \\
\hline & & 2 & 1870 & 1793 & 1750 & 4.401 & 4.589 & 4.702 & .2638 \\
\hline & & 3 & 1542 & 1791 & 1743 & 4.441 & 4.654 & 4.781 & 2495 \\
\hline
\end{tabular}

As in the first two examples, in the benchmark total output is decreasing in the amount of money. When $\lambda=\frac{11}{20}$, small increases in the amount of money are expansionary relative to the benchmark. That is, $Y_{12} / Y_{11}$ and $Y_{23} / Y_{22}$ are each larger than their values for the benchmark. However, as promised, a large increase is contractionary relative to the benchmark. That is, $Y_{13} / Y_{11}$ is smaller than its value in the benchmark. As in the other examples, the price level is less responsive to changes in the amount of money than it is in the benchmark. Again, welfare is lower with uninformed people than when everyone is informed.

\footnotetext{
${ }^{8}$ One force creating such monotonicity is the prevalence of meetings between informed producers and uninformed consumers, which depends on $\lambda$ in a non monotone way. In example 2, when there is always trade, output is minimal in such meetings because it satisfies the producer's individual rationality constraint for the smaller component of $\Delta$ no matter what is the current state. In example 3, this low output is partly overcome, but only at the cost of shutting down trade when the money supply increases.

${ }^{9}$ This is shown by comparing the value of $Z\left(y^{k}\right)=.1104$ (which is the same for each $k$, by the symmetry of the example) with the value of $Z$ in the table; note that $\Delta_{1}>\Delta_{2}>\Delta_{3}$, so that the mechanism that sets $a(0,3,1)=0$ satisfies producer truth-telling. Also, consumer individual rationality constraints hold because $u\left(\beta \Delta_{3}\right)>\beta \Delta_{1}$. Therefore, the value of $Z$ is a valid choice for $Z_{\min }$.
} 


\section{Randomization}

The mechanisms studied above are deterministic. More general mechanisms would permit randomization. There are three conceivable kinds of randomization. First, there could be randomization over outcomes, some of which include positive production and no transfer of money from the consumer to the producer. Such randomization would not satisfy our individual-rationality constraints, because the producer would not accept an outcome that calls for production and no transfer of money. ${ }^{10}$ Second, there could be randomization over outcomes in which each of several different amounts of production exchanges for a unit of money. While such randomization could be incentive feasible, we doubt that it could be optimal. We suspect that shifting probability toward the highest output that occurs with positive probability would be incentive feasible and would raise ex ante welfare. Third, there could be randomization over trade - no-trade sets. This kind of randomization is also incentive feasible and, as we show by example, can be optimal. We first present the example and then comment more generally on such randomization.

The reasoning behind the example is as follows. Consider the $S=2$ symmetric $\Pi$ given by (4.11). By proposition 3 and our examples, there is a critical value of $\varepsilon$ at which the optimum switches from $S^{p}(1)=\{1\}$, trade is shut down when the money supply increases, to $S^{p}(1)=\{1,2\}$, always-trade. At that point, the mechanism varies discontinuously, but $Z$ does not. Randomization over whether trade occurs could smooth the mechanism and, as we now explain, is likely to raise $Z$. For $q \in[0,1]$, let $a(0,2,1)=1$ with probability $q, y(0,2,1)=\beta \Delta_{2}$, and $y(0,1,1)=(1-q) \beta \Delta_{1}+q \beta \Delta_{2}$. For $q=0$, this corresponds to $S^{p}(1)=\{1\}$, while for $q=1$, it corresponds to $S^{p}(1)=\{1,2\}$. Also, if $\Delta_{1} \geq \Delta_{2}$, then it satisfies truth-telling for the informed producer. The part of $Z$ that depends directly on $q$ is proportional to

$$
(1-\varepsilon) \theta_{11} z\left[(1-q) \beta \Delta_{1}+q \beta \Delta_{2}\right]+\varepsilon \theta_{21} q z\left(\beta \Delta_{2}\right) .
$$

Holding $\Delta$ fixed, this term is strictly concave in $q$. Therefore, if this term has the same value at $q=0$ and at $q=1$-which, by proposition 2 , happens at the critical value of $\varepsilon$ - then it takes higher values at interior values of $q$. Although suggestive, this conclusion is not decisive because it ignores the dependence of $\Delta$ on $q$. Therefore, we produce an example which takes such dependence into account.

The example is the same as the $S=2$ examples in section 5 , except that $\lambda=\frac{1}{2}$ and $\varepsilon=\frac{1}{200}$. This $\varepsilon$ is very near the critical one at which the optimum switches from $S^{p}(1)=\{1\}$ to $S^{p}(1)=\{1,2\}$. Letting $Z(q)$ denote ex ante welfare as a function of $q$, we find $Z(1)=.1322575, Z(0)=.1322572$, and $Z\left(\frac{1}{2}\right)=.1322645$. Therefore, always-trade, $q=1$, is slightly better than shutting down trade, $q=0$, but mixing with $q=\frac{1}{2}$ is still better. It follows that randomization is optimal in this example.

Despite this finding, we suspect that randomization would not drastically change the kind of necessary conditions we found and, therefore, the kind of possibilities for the optimum that we displayed in section 5. Although we have not carried out a complete analysis, our suspicion is based on the following conjectures. Allowing

\footnotetext{
${ }^{10}$ See [2] for the study of such randomization in a similar model without uncertainty, but in which it is assumed that people can commit to the outcome of randomization.
} 
randomization changes the individual-rationality constraints and the expression for ex ante welfare in relatively simple ways. Therefore, for the relaxed welfare problem in which we drop the producer's truth-telling constraint, we suspect that there are necessary conditions for an optimum that are very much like the conclusions in proposition 1 and that will not imply randomization except near critical values for the deterministic problem. If the mechanism that produces the highest value of ex ante welfare for that relaxed problem turns out to satisfy truth-telling for the informed producer, then that mechanism is the optimum. Based on our section 4 discussion for $S=2$, we suspect that the solution to the relaxed problem is likely to satisfy producer truth-telling for many specifications in the region of the parameter space we study.

\section{Take-it-or-leave-it offers by consumers}

Matching models of the sort we are studying have often been analyzed using a bargaining rule. Among the most commonly used, because it is simple, is take-it-or-leave-it offers by consumers: the consumers says "I will give you my unit of money if and only if you produce the amount $x . "$ Our optimum resembles an equilibrium under such bargaining because, according to proposition 1, producers are being pushed to where they are on the margin between accepting and rejecting trade. In fact, any equilibrium under deterministic take-it-or-leave-it offers by consumers is one of the candidates for an optimum, one of the mechanisms that satisfy proposition 1. However, the optimum is not always such an equilibrium. In particular, at an optimum, an uninformed consumer facing an informed producer and making a take-it-or-leaveit offer leans toward an $x$ that shuts down trade in more states than the optimum dictates.

This tendency is easy to see if, for the moment, we ignore the dependence of $\Delta$ on the $S^{p}(i)$ sets. Then, if $\Delta_{j}>\Delta_{j+1}$, the ex ante welfare gain from shutting down trade in one additional state, $r$, when the previous state is $i$ is proportional to

$$
z\left(\beta \Delta_{r-1}\right) \sum_{j=1}^{r-1} \pi_{i j} \theta_{j i}-z\left(\beta \Delta_{r}\right) \sum_{j=1}^{r} \pi_{i j} \theta_{j i} \equiv B_{p}(r, i) .
$$

The difference here represents a trade-off between the output $\beta \Delta_{r-1}$ with some probability and the lower output $\beta \Delta_{r}$ with a higher probability. In contrast, the net gain to the consumer from demanding the output $\beta \Delta_{r-1}$ rather than the smaller output $\beta \Delta_{r}$ is proportional to

$$
\begin{gathered}
\sum_{j=1}^{r-1} \pi_{i j} \theta_{j i}\left[u\left(\beta \Delta_{r-1}\right)-\beta \Delta_{j}\right]-\sum_{j=1}^{r} \pi_{i j} \theta_{j i}\left[u\left(\beta \Delta_{r}\right)-\beta \Delta_{j}\right]= \\
B_{p}(r, i)+\beta\left(\Delta_{r-1}-\Delta_{r}\right) \sum_{j=1}^{r-1} \pi_{i j} \theta_{j i}
\end{gathered}
$$


Thus, the consumer sees an additional benefit to shutting down trade; by making the more demanding offer, the consumer avoids giving up money in state $r$ when it has a value that would command a higher output in state $r-1$. That term does not affect ex ante welfare, because the distribution of money at the start of the next period is not affected by whether trade occurs in state $r$.

Of course, the above comparison is not the correct one because it ignores the dependence of $\Delta$ on the $S^{p}(i)$ sets. Nevertheless, the comparison suggests that if the optimum has always-trade by a small margin, then that may not be an equilibrium under take-it-or-leave-it offers by consumers. That happens for the example presented in the discussion of randomization. There the optimum has always-trade and implies $\left(\Delta_{1}, \Delta_{2}\right)=(.2855514, .265532)$. If the uninformed consumer who meets an informed producer demands $\beta \Delta_{1}$ in exchange for surrendering money, then the consumer's expected gain is $\frac{199}{200}\left[\left(\beta \Delta_{1}\right)^{\frac{1}{2}}-\beta \Delta_{1}\right]$, where, recall, $\beta=\frac{27}{32}$. If, instead, the consumer demands $\beta \Delta_{2}$ in exchange for surrendering money, then the expected gain is $\left(\beta \Delta_{2}\right)^{\frac{1}{2}}-$ $\frac{199}{200} \beta \Delta_{1}-\frac{1}{200} \beta \Delta_{2}$. The difference is $\frac{199}{200}\left(\beta \Delta_{1}\right)^{\frac{1}{2}}-\left(\beta \Delta_{2}\right)^{\frac{1}{2}}+\frac{1}{200} \beta \Delta_{2}$, which is positive. Therefore, in this case, the optimum is not an equilibrium under take-it-or-leave-it offers by consumers.

As might be suspected, an equilibrium under take-it-or-leave-it offers by consumers may not exist unless there is randomization when the consumer is indifferent between two offers. In the above example, the $S^{p}(1)=\{1\}$ mechanism that satisfies proposition 1 is an equilibrium under take-it-or-leave-it offers by consumers. This is confirmed by showing that $\frac{199}{200}\left(\beta \Delta_{1}\right)^{\frac{1}{2}}-\left(\beta \Delta_{2}\right)^{\frac{1}{2}}+\frac{1}{200} \beta \Delta_{2} \geq 0$ when evaluated at the $\Delta$ implied by that mechanism - namely, $\left(\Delta_{1}, \Delta_{2}\right)=(.294211, .265728)$.

\section{Concluding Remarks}

Our analytical results and examples demonstrate that the existence of uninformed people is, indeed, important in the model. The ex ante optimum with uninformed people tends to display price level stickiness relative to the optimum for the same model without uninformed people, the benchmark. Also, impacts effects on output, relative to the benchmark, are sensitive to the distribution of money supply changes. In fact, such impact effects can be positive or negative and can be non monotone. Therefore, with uninformed people, even the best way of conducting trade in our model implies that aggregate output and the price level can behave in very complicated ways.

We are, of course, aware that models of output responses based on incomplete information about money-supply changes are widely viewed as less applicable in modern economies than for the economy that Hume was describing. That may be so, but there are two reasons for not dismissing such models. First, no model of output responses to money-supply changes seems free from dependence on technological developments that influence the spread of information. For example, it is hard to imagine that price-setting lags, no matter what accounts for them, stay fixed in response to developments like bar-coding that make it much easier to change and disseminate information about prices. Indeed, after all the attempts made by economists to spell out in a coherent way the kinds of claims that Hume made in the passage quoted 
in the introduction, the conclusion that seems warranted is that output responses to money supply changes seem dependent on messy details that cannot be expected to remain unchanged over long periods of time. Second, as we noted in the introduction, incomplete information about money-supply changes fits well with the assumption that there is incomplete public knowledge of individual histories, an assumption that is necessary for the essentiality of money. Given incomplete information about individual histories and decentralized trade, changes in the amount of money experienced by some people cannot be immediately deduced by everyone.

Finally, while we have been able to characterize an ex ante optimum in a class of deterministic mechanisms, we have done so only for a minimalist model —one that is rich enough to be interesting, yet simple enough to be tractable. In addition to considering randomization and other regions of the parameter space, it would be desirable to check the robustness of our findings to some of our more extreme assumptions. We have made substantial use of the indivisibility of money and the unit upper bound. Obviously, truth-telling constraints would not have such simple implications if the money trades could be other than surrendering 0 or 1 unit. Settings with richer individual holdings of money would be much harder to study because in them the distribution of money holdings depends on the mechanism. Another extension to consider is an information structure in which the identity of the informed is not common knowledge. Such a change gives rise to additional truth-telling constraints and to two-sided asymmetric information in meetings. A third extension would consider lengthening the lag with which there is a public announcement of the state. Such a version would permit the study of how knowledge of states spread through the meeting process. At a minimum, such lengthening leads to a proliferation of aggregate and individual information states. 


\section{Appendix}

This section contains proofs of the lemmas and propositions.

Lemma 1. If (2.1) holds, then $\theta_{j i}$ and $\lambda-\theta_{j i}$ are non-negative.

Proof. We have $\left(1-m_{i}\right) \lambda \geq\left(1-m_{S}\right) \lambda \geq m_{S} \lambda \geq m_{S}-m_{1} \geq m_{j}-m_{i}$, where the second inequality follows from the first part of (2.1) and the third from the lower bound condition on $\lambda$ in (2.1). This implies that $\theta_{j i} \geq 0$. Also, $(1-\lambda) m_{i} \leq(1-\lambda) m_{S}=$ $m_{S}-\lambda m_{S} \leq m_{S}-\left(m_{S}-m_{1}\right) \leq m_{j}$, where the second inequality follows from the lower bound condition on $\lambda$. in (2.1). This implies that $m_{j} \geq(1-\lambda) m_{i}$, and, hence, that $\lambda-\theta_{j i}$, is non-negative.

Lemma 2. For any mechanism, $Z$ satisfies (3.16).

Proof. From the definition of $\eta_{i j}$ (see (3.5)),

$$
m_{j}-m_{i}= \begin{cases}-m_{i} \eta_{i j} & j<i \\ \left(1-m_{i}\right) \eta_{i j} & \text { otherwise. }\end{cases}
$$

Then, if we substitute (3.4) into (3.15), we obtain

$$
\begin{aligned}
Z= & \sum_{i=1}^{S} p_{i}\left[m_{i} V_{1}(i)+\left(1-m_{i}\right) V_{0}(i)\right] \\
= & \sum_{i=1}^{S} \sum_{j=1}^{S} p_{i} \pi_{i j}(1-\lambda)\left[m_{i} v_{1}(0, j, i)+\left(1-m_{i}\right) v_{0}(0, j, i)\right] \\
& +\sum_{i=1}^{S} \sum_{j=1}^{S} p_{i} \pi_{i j} \lambda\left[m_{i} v_{1}(1, j, i)+\left(1-m_{i}\right) v_{0}(1, j, i)\right] \\
& +\sum_{i=1}^{S} \sum_{j=1}^{S} p_{i} \pi_{i j}\left(m_{j}-m_{i}\right)\left[v_{1}(1, j, i)-v_{0}(1, j, i)\right] .
\end{aligned}
$$

Therefore,

$$
\begin{aligned}
Z= & \sum_{i=1}^{S} \sum_{j=1}^{S} p_{i} \pi_{i j}(1-\lambda)\left[m_{i} v_{1}(0, j, i)+\left(1-m_{i}\right) v_{0}(0, j, i)\right] \\
& +\sum_{i=1}^{S} \sum_{j=1}^{S} p_{i} \pi_{i j}\left[\left(\lambda-\theta_{j i}\right) v_{1}(1, j, i)+\theta_{j i} v_{0}(1, j, i)\right] .
\end{aligned}
$$

Using (3.3), we have

$Z=\beta \sum_{i=1}^{S} \sum_{j=1}^{S} p_{i} \pi_{i j}(1-\lambda)\left[m_{i} V_{1}(j)+\left(1-m_{i}\right) V_{0}(j)\right]$ 


$$
\begin{aligned}
& +\beta \sum_{i=1}^{S} \sum_{j=1}^{S} p_{i} \pi_{i j}\left[\left(\lambda-\theta_{j i}\right) V_{1}(j)+\theta_{j i} V_{0}(j)\right] \\
& +\frac{1}{N} \sum_{i=1}^{S} \sum_{j=1}^{S} p_{i} \pi_{i j}(1-\lambda) m_{i}\left[\theta_{j i} G_{1}(0,1, j, i)+(1-\lambda)\left(1-m_{i}\right) G_{1}(0,0, j, i)\right] \\
& +\frac{1}{N} \sum_{i=1}^{S} \sum_{j=1}^{S} p_{i} \pi_{i j}(1-\lambda)\left(1-m_{i}\right)\left[\left(\lambda-\theta_{j i}\right) G_{0}(1,0, j, i)+(1-\lambda) m_{i} G_{0}(0,0, j, i)\right] \\
& +\frac{1}{N} \sum_{i=1}^{S} \sum_{j=1}^{S} p_{i} \pi_{i j}\left(\lambda-\theta_{j i}\right)\left[\theta_{j i} G_{1}(1,1, j, i)+(1-\lambda)\left(1-m_{i}\right) G_{1}(1,0, j, i)\right] \\
& +\frac{1}{N} \sum_{i=1}^{S} \sum_{j=1}^{S} p_{i} \pi_{i j} \theta_{j i}\left[\left(\lambda-\theta_{j i}\right) G_{0}(1,1, j, i)+(1-\lambda) m_{i} G_{0}(0,1, j, i)\right] .
\end{aligned}
$$

Then, using (3.2), this expression can be written as

$$
\begin{aligned}
Z= & \beta \sum_{i=1}^{S} \sum_{j=1}^{S} p_{i} \pi_{i j}\left[m_{j} V_{1}(j)+\left(1-m_{j}\right) V_{0}(j)\right]+ \\
& \frac{1}{N} \sum_{i=1}^{S} \sum_{j=1}^{S} p_{i} \pi_{i j}(1-\lambda) m_{i}\left[\theta_{j i} z(y(0, j, i))+(1-\lambda)\left(1-m_{i}\right) z(y(0,0, i))\right]+ \\
& \frac{1}{N} \sum_{i=1}^{S} \sum_{j=1}^{S} p_{i} \pi_{i j}\left(\lambda-\theta_{j i}\right)\left[\theta_{j i} z(y(j, j, i))+(1-\lambda)\left(1-m_{i}\right) z(y(j, 0, i))\right] .(9.5)
\end{aligned}
$$

Finally, using the definition of the invariant probabilities, the $p_{i}$,

$$
\begin{gathered}
\sum_{i=1}^{S} \sum_{j=1}^{S} p_{i} \pi_{i j}\left[m_{j} V_{1}(j)+\left(1-m_{j}\right) V_{0}(j)\right]= \\
\sum_{j=1}^{S}\left[m_{j} V_{1}(j)+\left(1-m_{j}\right) V_{0}(j)\right] \sum_{i=1}^{S} p_{i} \pi_{i j}= \\
\sum_{j=1}^{S} p_{j}\left[m_{j} V_{1}(j)+\left(1-m_{j}\right) V_{0}(j)\right]=Z
\end{gathered}
$$

When this is substituted into (9.5), we get (3.16), as required.

Lemma 3. If $(y, a)$ is incentive feasible, then $\Delta(y, a)$ satisfies,

$$
\frac{1}{\gamma_{i}^{1}\left(S^{c}(i)\right)} \sum_{j \in S^{c}(i)} \pi_{i j}\left(\lambda-\theta_{j i}\right) \beta \Delta_{j} \leq \frac{1}{\gamma_{i}^{1}\left(\mathbb{S}_{+}\right)} \sum_{j \in \mathbb{S}_{+}} \pi_{i j}\left(\lambda-\theta_{j i}\right) \beta \Delta_{j} .
$$


Proof. We have

$$
\begin{aligned}
D & \equiv \frac{\beta}{\gamma_{i}^{1}\left(\mathbb{S}_{+}\right)} \sum_{j \in \mathbb{S}_{+}} \pi_{i j}\left(\lambda-\theta_{j i}\right) \Delta_{j}-\frac{\beta}{\gamma_{i}^{1}\left(S^{c}(i)\right)} \sum_{j \in S^{c}(i)} \pi_{i j}\left(\lambda-\theta_{j i}\right) \Delta_{j} \\
& =\frac{\beta\left(\sigma-\sigma^{\prime}\right)}{\sigma \sigma^{\prime}} \sum_{j \in S^{c}(i)} \pi_{i j}\left(\lambda-\theta_{j i}\right) \Delta_{j}+\frac{\beta}{\sigma^{\prime}} \sum_{j \in \mathbf{S}_{+}-S^{c}(i)} \pi_{i j}\left(\lambda-\theta_{j i}\right) \Delta_{j},
\end{aligned}
$$

where $\sigma \equiv \gamma_{i}^{1}\left(S^{c}(i)\right) \leq \sigma^{\prime} \equiv \gamma_{i}^{1}\left(\mathbb{S}_{+}\right)$. Now, let $x=\min _{j \in \mathbb{S}_{+}-S^{c}(i)} \Delta_{j}$. Then, by (3.12),

$$
D \geq \frac{\beta\left(\sigma-\sigma^{\prime}\right)}{\sigma^{\prime}} x+\frac{\beta}{\sigma^{\prime}}\left(\sigma^{\prime}-\sigma\right) x=0
$$

as required.

Lemma 4. Let

$$
h(x ; y, a) \equiv b(y)+\beta C(a) x \text { for } x \in R^{S} .
$$

Then, (i) $\Delta(y, a)$ is a fixed point of $h(\cdot ; y, a)$ and vice versa; (ii) $h(\cdot ; y, a)$ satisfies Blackwell's sufficient conditions for contraction; (iii) if $x \geq \Delta(y, a)$ and $h\left(x ; y^{\prime}, a^{\prime}\right) \geq$ $\Delta(y, a)$, then $\Delta\left(y^{\prime}, a^{\prime}\right) \geq \Delta(y, a)$; and (iv) if $y^{\prime} \geq y$, then $\Delta\left(y^{\prime}, a\right) \geq \Delta(y, a)$.

Proof. From (3.4), we have

$$
\Delta_{i}=\sum_{j=1}^{S} \pi_{i j}\left[(1-\lambda) \delta_{i}(0, j)+\left(\lambda-\eta_{i j}\right) \delta_{i}(1, j)\right],
$$

where $\delta_{i}(\iota, j) \equiv v_{1}(\iota, j, i)-v_{1}(\iota, j, i)$. Also, from (3.3), we have

$$
\begin{aligned}
N \delta_{i}(\iota, j)= & N \beta \Delta_{j}+\theta_{j i} G_{1}(\iota, 1, j, i)+(1-\lambda)\left(1-m_{i}\right) G_{1}(\iota, 0, j, i) \\
& -\left(\lambda-\theta_{j i}\right) G_{0}(1, \iota, j, i)-(1-\lambda) m_{i} G_{0}(0, \iota, j, i) .
\end{aligned}
$$

Then, if we substitute from (9.12) into (9.11) and use the definition of $G_{k}\left(\iota^{c}, \iota^{p}, j, i\right)$, it follows that $\Delta(y, a)$ satisfies the matrix equation,

$$
\Delta(y, a)=b(y)+\beta C(a) \Delta(y, a) .
$$

By the definition of $h(\cdot ; y, a)$, it follows that $\Delta(y, a)$ is a fixed points of $h$. Therefore, (i) is true.

As regards (ii), it follows from (4.5) that $c_{i j}(a) / \pi_{i j} \in\left(1-\eta_{i j}\right)\left[1-N^{-1}, 1\right]$, where the lower endpoint is attained when money is always transferred and the upper endpoint when money is never transferred. Because (2.1) implies that $\eta_{i j}<1$, it follows that $c_{i j} / \pi_{i j} \in(0,1]$. That implies that $h(\cdot ; y, a)$ is monotone increasing and satisfies discounting, Blackwell's sufficient conditions for contraction.

Claim (iii) is an obvious consequence of contraction. That is, if $h^{(n)}\left(\Delta(y, a) ; y^{\prime}, a^{\prime}\right) \geq$ $\Delta(y, a)$, then $h^{(n+1)}\left(\Delta(y, a) ; y^{\prime}, a^{\prime}\right)=h\left[h^{(n)}\left(\Delta(y, a) ; y^{\prime}, a^{\prime}\right) ; y^{\prime}, a^{\prime}\right] \geq h\left(\Delta(y, a) ; y^{\prime}, a^{\prime}\right) \geq$ 
$\Delta(y, a)$, where $h^{(n)}\left(x ; y^{\prime}, a^{\prime}\right)$ is the $n$-th iterate of $h$ applied to $x$, where the first inequality follows from monotonicity of $h$ and the second inequality is the hypothesis, which serves as the initial condition for the induction step. But by the contraction property, $\lim _{n \rightarrow \infty} h^{(n)}\left(x ; y^{\prime}, a^{\prime}\right)=\Delta\left(y^{\prime}, a^{\prime}\right)$, which implies $\Delta\left(y^{\prime}, a^{\prime}\right) \geq \Delta(y, a)$.

From (4.6), $b(y)$ is increasing in $y$. That and claim (iii) imply claim (iv).

Lemma 5. If $(y, a)$ satisfies producer individual-rationality constraints and has constant output over trade states in meetings between informed and uninformed, then $y\left(s, s^{\prime}, i\right) \leq \beta \max _{i} \Delta_{i}(y, a) \leq y_{\max }^{1}$.

Proof. Let $K=\max _{i} \Delta_{i}(y, a)$. Then $y\left(s, s^{\prime}, i\right) \leq a\left(s, s^{\prime}, i\right) \beta K$, for all $s, s^{\prime}, i$.

From equations 3.3 we have

$$
\begin{aligned}
N \delta_{i}(0, j)= & N \beta \Delta_{j}+\theta_{j i} z(y(0, j, i))+(1-\lambda)\left(1-m_{i}\right) z(y(0,0, i)) \\
& +(1-\lambda)\left[y(0,0, i)-a(0,0, i) \beta \Delta_{j}\right]+\theta_{j i}\left[y(0, j, i)-a(0, j, i) \beta \Delta_{j}\right] \\
& +\left(\lambda-\theta_{j i}\right)\left[y(j, 0, i)-a(j, 0, i) \beta \Delta_{j}\right] \\
N \delta_{i}(1, j)= & N \beta \Delta_{j}+\theta_{j i} z(y(j, j, i))+(1-\lambda)\left(1-m_{i}\right) z(y(j, 0, i)) \\
& +\lambda\left[y(j, j, i)-a(j, j, i) \beta \Delta_{j}\right]+(1-\lambda) m_{i}\left[y(0, j, i)-a(0, j, i) \beta \Delta_{j}\right] \\
& +(1-\lambda)\left(1-m_{i}\right)\left[y(j, 0, i)-a(j, 0, i) \beta \Delta_{j}\right]
\end{aligned}
$$

where $\Delta_{j}=\Delta_{j}(y, a)$.

Let $\zeta=\max _{s, s^{\prime}, i} z\left(y\left(s, s^{\prime}, i\right)\right)$. Then, using the definition of $\theta$ and the producer's individual-rationality constraints,

$$
\begin{aligned}
N \delta_{i}(0, j) \leq & N \beta \Delta_{j}+\left(1-m_{j}\right) \zeta+(1-\lambda)\left[y(0,0, i)-a(0,0, i) \beta \Delta_{j}\right] \\
& +\left(\lambda-\theta_{j i}\right)\left[y(j, 0, i)-a(j, 0, i) \beta \Delta_{j}\right] \\
N \delta_{i}(1, j) \leq & N \beta \Delta_{j}+\left(1-m_{j}\right) \zeta \\
& +(1-\lambda)\left(1-m_{i}\right)\left[y(j, 0, i)-a(j, 0, i) \beta \Delta_{j}\right]
\end{aligned}
$$

These inequalities can be rewritten as

$$
\begin{aligned}
N \delta_{i}(0, j) \leq & \left(1-m_{j}\right) \zeta+(1-\lambda) y(0,0, i)+\left(\lambda-\theta_{j i}\right) y(j, 0, i) \\
& +\left[N-(1-\lambda) a(0,0, i)-\left(\lambda-\theta_{j i}\right) a(j, 0, i)\right] \beta \Delta_{j} \\
N \delta_{i}(1, j) \leq & \left(1-m_{j}\right) \zeta+(1-\lambda)\left(1-m_{i}\right) y(j, 0, i) \\
& +\left[N-(1-\lambda)\left(1-m_{i}\right) a(j, 0, i)\right] \beta \Delta_{j}
\end{aligned}
$$

Then since the coefficients of $\beta \Delta_{j}$ are positive,

$$
\begin{aligned}
N \delta_{i}(0, j) \leq & \left(1-m_{j}\right) \zeta+(1-\lambda) y(0,0, i)+\left(\lambda-\theta_{j i}\right) y(j, 0, i) \\
& +\left[N-(1-\lambda) a(0,0, i)-\left(\lambda-\theta_{j i}\right) a(j, 0, i)\right] \beta K \\
= & N \beta K+\left(1-m_{j}\right) \zeta+(1-\lambda)[y(0,0, i)-a(0,0, i) \beta K] \\
& +\left(\lambda-\theta_{j i}\right)[y(j, 0, i)-a(j, 0, i) \beta K]
\end{aligned}
$$




$$
\begin{aligned}
N \delta_{i}(1, j) \leq & \left(1-m_{j}\right) \zeta+(1-\lambda)\left(1-m_{i}\right) y(j, 0, i) \\
& +\left[N-(1-\lambda)\left(1-m_{i}\right) a(j, 0, i)\right] \beta K \\
= & N \beta K+\left(1-m_{j}\right) \zeta \\
& +(1-\lambda)\left(1-m_{i}\right)[y(j, 0, i)-a(j, 0, i) \beta K]
\end{aligned}
$$

But then since $y(0,0, i) \leq a(0,0, i) \beta K$, and $y(j, 0, i) \leq a(j, 0, i) \beta K$,

$$
\begin{aligned}
& N \delta_{i}(0, j) \leq N \beta K+\left(1-m_{j}\right) \zeta \\
& N \delta_{i}(1, j) \leq N \beta K+\left(1-m_{j}\right) \zeta
\end{aligned}
$$

Now from (9.11), we have

$$
\begin{aligned}
\Delta_{i} & =\sum_{j=1}^{S} \pi_{i j}\left[(1-\lambda) \delta_{i}(0, j)+\left(\lambda-\eta_{i j}\right) \delta_{i}(1, j)\right], \\
& \leq \sum_{j=1}^{S} \pi_{i j}\left(1-\eta_{i j}\right)\left[\beta K+\frac{1}{N}\left(1-m_{j}\right) \zeta\right]
\end{aligned}
$$

Then because this holds for all $i$, and $\sum_{j} \pi_{i j}\left(1-\eta_{i j}\right) \leq 1$, and $1-m_{j} \leq 1-m_{1}$,

$$
K \leq \frac{\zeta\left(1-m_{1}\right)}{N(1-\beta)}
$$

By the definition of $y_{\max }^{1}$ and the assumption $y^{*} \geq y_{\max }^{1}$, we have

$$
\frac{\beta z\left(y^{*}\right)\left(1-m_{1}\right)}{N(1-\beta)} \leq y^{*} .
$$

Then since $\zeta \leq z\left(y^{*}\right)$ it follows that

$$
\beta K \leq \frac{\beta z\left(y^{*}\right)\left(1-m_{1}\right)}{N(1-\beta)} \leq y^{*} .
$$

Now because the function $z$ is increasing on the interval $\left[0, y^{*}\right]$ and because $y\left(s, s^{\prime}, i\right) \leq \beta K$, it follows that $\zeta \leq z(\beta K)$. Thus (9.20) implies

$$
\beta K \leq \frac{\beta z(\beta K)\left(1-m_{1}\right)}{N(1-\beta)} .
$$

This, in turn, implies $\beta K \leq y_{\max }^{1}$.

Proposition 1. If $(y, a)$ is optimal and satisfies $u\left(\beta \min _{s} \Delta_{s}(y, a)\right) \geq y_{\max }^{1}$, then (i) $S^{c}(i) \equiv \mathbb{S}_{+}$and $(y, a)$ satisfies (4.7)-(4.10); (ii) for each $i, S^{p}(i)$ is not empty; and (iii) if $\Delta_{j}(y, a)=\Delta_{k}(y, a)$, then for each $i$, either $j, k \in S^{p}(i)$ or $j, k \in \mathbb{S}_{+}-S^{p}(i)$. 
Proof. Suppose that $(y, a)$ is optimal, but does not satisfy (i). Then we consider $\left(y^{\prime}, a^{\prime}\right)$ given by (4.7)-(4.10), but with $\Delta(y, a)$ inserted on the right-hand sides of (4.7)-(4.10) and with $S^{c}(i)$ replaced by $\mathbb{S}_{+}$.

Our first task is to show that $y^{\prime} \geq y$. For all but, $Y^{c}(i)^{\prime}$, this follows immediately from the fact that each component of $y$ satisfies the relevant producer individual rationality constraint. For $Y^{c}(i)^{\prime}$, we use lemma 3 because we are replacing a somewhat arbitrary $S^{c}(i)$ set with $\mathbb{S}_{+}$. We have,

$$
Y^{c}(i) \leq \frac{1}{\gamma_{i}^{1}\left(S^{c}(i)\right)} \sum_{j \in S^{c}(i)} \pi_{i j}\left(\lambda-\theta_{j i}\right) \beta \Delta_{j} \leq \frac{1}{\gamma_{i}^{1}\left(\mathbb{S}_{+}\right)} \sum_{j \in \mathbb{S}_{+}} \pi_{i j}\left(\lambda-\theta_{j i}\right) \beta \Delta_{j}=Y^{c}(i)^{\prime},
$$

where the first inequality is by incentive feasibility of $y$ and where the second is lemma 3 .

We next show that $\Delta\left(y^{\prime}, a^{\prime}\right) \geq \Delta(y, a)$. By the third part of lemma 4 , it is enough to show that $h_{i}\left(\Delta(y, a) ; y^{\prime}, a^{\prime}\right) \geq \Delta_{i}(y, a)=h_{i}(\Delta(y, a) ; y, a)$, where $h_{i}$ is the $i$-th component of the mapping defined in (4.4). Because $h_{i}$ is a sum of terms, we can deal one-at-a-time with the replacements given by (4.7)-(4.10). Because $y^{\prime} \geq y$ and $h(x ; y, a)$ is increasing in $y$, we need only concern ourselves with situations in which the replacement of $(y, a)$ by $\left(y^{\prime}, a^{\prime}\right)$ involves replacing a no-trade outcome by a trade outcome.

An informed consumer meets an uninformed producer, (4.7).

Considering only the (4.7) substitution, (9.13) implies that

$$
\begin{aligned}
& \quad \frac{N}{1-\lambda}\left[h_{i}\left(\Delta(y, a) ; y^{\prime}, a^{\prime}\right)-h_{i}(\Delta(y, a) ; y, a)\right]= \\
& \left(1-m_{i}\right) \sum_{j \in S^{c}(i)} \pi_{i j}\left(\lambda-\eta_{i j}\right)\left[u\left(Y^{c}(i)^{\prime}\right)-\beta \Delta_{j}\right]+\sum_{j \in S^{c}(i)} \pi_{i j}\left(\lambda-\theta_{i j}\right)\left[Y^{c}(i)^{\prime}-\beta \Delta_{j}\right]+ \\
& \left(1-m_{i}\right) \sum_{j \in \mathbb{S}_{+}-S^{c}(i)} \pi_{i j}\left(\lambda-\eta_{i j}\right)\left[u\left(Y^{c}(i)^{\prime}\right)-\beta \Delta_{j}\right]+\sum_{j \in \mathbb{S}_{+}-S^{c}(i)} \pi_{i j}\left(\lambda-\theta_{i j}\right)\left[Y^{c}(i)^{\prime}-\beta \Delta_{j}\right] \\
& \quad-\left(1-m_{i}\right) \sum_{j \in S^{c}(i)} \pi_{i j}\left(\lambda-\eta_{i j}\right)\left[u\left(Y^{c}(i)\right)-\beta \Delta_{j}\right]-\sum_{j \in S^{c}(i)} \pi_{i j}\left(\lambda-\theta_{i j}\right)\left[Y^{c}(i)-\beta \Delta_{j}\right] \\
& =\left(1-m_{i}\right) \sum_{j \in S^{c}(i)} \pi_{i j}\left(\lambda-\eta_{i j}\right)\left[u\left(Y^{c}(i)^{\prime}\right)-u\left(Y^{c}(i)\right)\right]+\pi_{j \in \mathbb{S}_{+}-S^{c}(i)} \pi_{i j}\left(\lambda-\eta_{i j}\right)\left[u\left(Y^{c}(i)^{\prime}\right)-\beta \Delta_{j}\right]-\sum_{j \in S^{c}(i)} \pi_{i j}\left(\lambda-\theta_{i j}\right)\left[Y^{c}(i)-\beta \Delta_{j}\right]
\end{aligned}
$$

where the second equality follows from the definition of $Y^{c}(i)^{\prime}$. Because $(y, a)$ is incentive-feasible and, in particular, satisfies the uninformed producer's individualrationality constraint, we have $\sum_{j \in S^{c}(i)} \pi_{i j}\left(\lambda-\theta_{i j}\right)\left[Y^{c}(i)-\beta \Delta_{j}\right] \leq 0$. Therefore,

$$
\frac{N}{(1-\lambda)\left(1-m_{i}\right)}\left[h_{i}\left(\Delta(y, a) ; y^{\prime}, a^{\prime}\right)-h_{i}(\Delta(y, a) ; y, a)\right] \geq
$$




$$
\sum_{j \in S^{c}(i)} \pi_{i j}\left(\lambda-\eta_{i j}\right)\left[u\left(Y^{c}(i)^{\prime}\right)-u\left(Y^{c}(i)\right)\right]+\sum_{j \in \mathbb{S}_{+}-S^{c}(i)} \pi_{i j}\left(\lambda-\eta_{i j}\right)\left[u\left(Y^{c}(i)^{\prime}\right)-\beta \Delta_{j}\right]
$$

The first term is nonnegative by (9.24). The second term is nonnegative because $Y^{c}(i)^{\prime} \geq \beta \min _{s} \Delta_{s}$ and $u\left(\beta \min _{s} \Delta_{s}\right) \geq y_{\max }^{1} \geq \beta \max _{s} \Delta_{s}$ by hypothesis and lemma 4.

An uninformed consumer meets an uninformed producer, (4.8).

Here, the replacement of no trade by trade given by (4.8) implies, according to (9.13), that

$$
\begin{gathered}
N\left[h_{i}\left(\Delta(y, a) ; y^{\prime}, a^{\prime}\right)-h_{i}(\Delta(y, a) ; y, a)\right]= \\
(1-\lambda)^{2}\left(1-m_{i}\right) \sum_{j=1}^{S} \pi_{i j}\left[u\left(y(0,0, i)^{\prime}\right)-\beta \Delta_{j}\right]+(1-\lambda) m_{i} \sum_{j=1}^{S} \pi_{i j}\left[y(0,0, i)^{\prime}-\beta \Delta_{j}\right]= \\
(1-\lambda)^{2}\left(1-m_{i}\right) \sum_{j=1}^{S} \pi_{i j}\left[u\left(y(0,0, i)^{\prime}\right)-\beta \Delta_{j}\right] \geq 0 .
\end{gathered}
$$

The second equality follows from the definition of $y(0,0, i)^{\prime}$ and the inequality is implied by $y(0,0, i)^{\prime} \geq \beta \min _{s} \Delta_{s}$ and $u\left(\beta \min _{s} \Delta_{s}\right) \geq y_{\max }^{1} \geq \beta \max _{s} \Delta_{s}$.

An informed consumer meets an informed producer, (4.9).

Here, the replacement of no trade by trade given by (4.9) implies, according to (9.13), that

$$
N\left[h_{i}\left(\Delta(y, a) ; y^{\prime}, a^{\prime}\right)-h_{i}(\Delta(y, a) ; y, a)\right]=\sum_{j} \pi_{i j}\left(\lambda-\eta_{i j}\right) \theta_{j i}\left[u\left(\beta \Delta_{j}\right)-\beta \Delta_{j}\right] \geq 0,
$$

where the summation is over some set of current states.

We now have $y^{\prime} \geq y$ and $\Delta\left(y^{\prime}, a^{\prime}\right) \geq \Delta(y, a)$. It follows that $\left(y^{\prime}, a^{\prime}\right)$ satisfies all the producer individual-rationality constraints. Moreover, by lemma $5, \Delta\left(y^{\prime}, a^{\prime}\right) \leq y_{\max }^{1}$. Therefore, $u\left(\beta \min _{s} \Delta_{s}\left(y^{\prime}, a^{\prime}\right)\right) \geq u\left(\beta \min _{s} \Delta_{s}(y, a)\right) \geq y_{\max }^{1} \geq \beta \max _{s} \Delta_{s}\left(y^{\prime}, a^{\prime}\right)$. This implies that $\left(y^{\prime}, a^{\prime}\right)$ satisfies all the consumer individual-rationality constraints. It also satisfies truth-telling for the informed consumer because it has trade in every state when the consumer is informed. But $\left(y^{\prime}, a^{\prime}\right)$ may or may not satisfy truth-telling for informed producers. If it does, then we have a contradiction because $y_{\max }^{1} \geq y^{\prime} \geq y$ and we have either increased output or replaced no-trade by trade, both of which increase $Z$.

If not, because $\Delta\left(y^{\prime}, a^{\prime}\right) \geq \Delta(y, a)$, then there is some previous state $i$ and some current state $r$ such that $a(0, r, i)=0$ and

$$
\beta \Delta_{r}\left(y^{\prime}, a^{\prime}\right)>Y^{p}(i)^{\prime} \geq \beta \Delta_{r}(y, a)
$$

where the second inequality holds because $(y, a)$ is incentive-feasible. That is, the informed producer would like to get money in additional states when responding to the incentives implied by $\Delta\left(y^{\prime}, a^{\prime}\right)$ as opposed to those implied by $\Delta(y, a)$. If so, then define a new mechanism $\left(y^{\prime \prime}, a^{\prime \prime}\right)$ that agrees with $\left(y^{\prime}, a^{\prime}\right)$ except that 


$$
a(0, r, i)^{\prime \prime}=1 \text { and } y(0, r, i)^{\prime \prime}=Y^{p}(i)^{\prime} .
$$

for all pairs $r, i$ satisfying (9.28). Note that this increases the number of states with trade when the producer has private information. The replacement of no-trade by trade in state $r$ implies, according to (9.13), that

$$
\begin{gathered}
\frac{N}{1-\lambda}\left[h_{i}\left(\Delta(y, a) ; y^{\prime \prime}, a^{\prime \prime}\right)-h_{i}(\Delta(y, a) ; y, a)\right]= \\
\pi_{i r} \theta_{r i}\left[u\left(Y^{p}(i)^{\prime}\right)-\beta \Delta_{r}(y, a)\right]+\pi_{i r}\left(\lambda-\eta_{i r}\right) m_{i}\left[Y^{p}(i)^{\prime}-\beta \Delta_{r}(y, a)\right] \geq 0 .
\end{gathered}
$$

Therefore, by the third part of lemma $4, \Delta\left(y^{\prime \prime}, a^{\prime \prime}\right) \geq \Delta(y, a)$. Thus (by repeating the arguments given above for $\left.\left(y^{\prime}, a^{\prime}\right)\right)$, the mechanism $\left(y^{\prime \prime}, a^{\prime \prime}\right)$ satisfies all the constraints except possibly the producer's truth-telling constraints. If the producer's truth-telling constraints are also satisfied then $\left(y^{\prime \prime}, a^{\prime \prime}\right)$ is incentive-feasible and has higher output and, therefore, higher welfare than $(y, a)$, contradicting the assumption that $(y, a)$ is optimal. But, again, it may happen that $\left(y^{\prime \prime}, a^{\prime \prime}\right)$ violates the producer's truth-telling constraint. If so, then we amend $\left(y^{\prime \prime}, a^{\prime \prime}\right)$ by adding states in which trade occurs. Since the producer's truth-telling constraints are satisfied if $S^{p}(i)=\mathbf{S}_{+}$, and since the number of states is finite, repetition of these steps must lead to a mechanism that satisfies the producer's truth-telling constraints, with higher output and therefore higher welfare than $(y, a)$, a contradiction. Thus, condition (i) is necessary for an optimum.

Now suppose $(y, a)$ is optimal, but $S^{p}(i)$ is empty. Let $k$ be such that $\Delta_{k}(y, a)=$ $\max _{j} \Delta_{j}(y, a)$. Then consider $\left(y^{\prime}, a^{\prime}\right)$ that agrees with $(y, a)$ except that $a(0, k, i)^{\prime}=1$ and $y(0, k, i)^{\prime}=\beta \Delta_{k}(y, a)$. Then, as was done above, it can be shown that $\Delta\left(y^{\prime}, a^{\prime}\right) \geq$ $\Delta(y, a)$. It follows, as in the argument above, that $\left(y^{\prime}, a^{\prime}\right)$ gives higher welfare and satisfies all the constraints except perhaps the truth-telling constraint for informed producers. However, if it fails to satisfy that constraint, then there are two possibilities. One is that (9.28) might hold with respect to a previous state $i_{0} \neq i$ and, therefore, such that $S^{p}\left(i_{0}\right)^{\prime}=S^{p}\left(i_{0}\right)$. In that case we can apply the argument above which leads to a contradiction. The other is that

$$
\beta \Delta_{r}\left(y^{\prime}, a^{\prime}\right)>y(0, k, i)^{\prime}=\beta \Delta_{k}(y, a) \geq \beta \Delta_{r}(y, a)
$$

where the last inequality holds because $\Delta_{k}(y, a)=\max _{j} \Delta_{j}(y, a)$. But this, too, is a version of (9.28). Hence, again, we can define a new mechanism $\left(y^{\prime \prime}, a^{\prime \prime}\right)$ that agrees with $\left(y^{\prime}, a^{\prime}\right)$ except that

$$
a(0, r, i)^{\prime \prime}=1 \text { and } y(0, r, i)^{\prime \prime}=\beta \Delta_{k}(y, a) .
$$

for all states $r$ satisfying (9.31). Thus, in this case also, we can apply the argument above which leads to a contradiction.

Finally, suppose $(y, a)$ is optimal but does not satisfy (iii). Then the optimum is such that $S^{p}(i)$ for some $i$ partitions states in such a way that there are two current states, say $j$ and $k$ with $\Delta_{j}=\Delta_{k}$ and $j \in S^{p}(i)$ and $k \notin S^{p}(i)$. By truth-telling, it follows that $\Delta_{j}=\min _{l \in S^{p}(i)} \Delta_{l}$. Therefore, by necessary condition (i), $(y, a)$ has 
$Y^{p}(i)=\beta \Delta_{j}$. Then amend $(y, a)$ by making the omitted state a trade state without changing output. The rest of the argument is exactly like the argument for necessary condition (i), and, therefore, produces a contradiction.

Lemma 6. Given $S^{p}(i)$ for each $i \in \mathbf{S}_{+}$, there exists a unique monetary mechanism that satisfies (4.7)-(4.10).

Proof. We will use the following result (see [7]):

Suppose $f=\left(f^{1}, f^{2}, \ldots, f^{n}\right)$ is a function from $R^{n}$ to $R^{n}$ such that

(i) $f$ is increasing

(ii) for each $i, f^{i}$ is a strictly concave function from $R^{n}$ to $R$

(iii) $f(0) \geq 0$

(iv) there is a positive vector $x^{a}$ such that $f\left(x^{a}\right)>x^{a}$

(v) there is a vector $x^{b}>x^{a}$ such that $f\left(x^{b}\right)<x^{b}$.

Then there exists a unique positive vector $x$ such that $f(x)=x$. Moreover, $x \in\left(x^{a}, x^{b}\right)$.

The proof proceeds by defining a mapping that satisfies conditions (i)-(v) and each of whose fixed points is a monetary mechanism that satisfies (4.7)-(4.10). Let $f$ from $R^{S}$ to $R^{S}$ be defined as follows. For $\Delta \in R^{S}$ let $y=g(\Delta)$ be the mapping defined by the equations

$$
\begin{aligned}
y(j, j, i) & =\beta \Delta_{j} \text { for all }(i, j) \in \mathbf{S}_{+} \times \mathbf{S}_{+}, \\
y(0,0, i) & =\beta \sum_{j=1}^{S} \pi_{i j} \Delta_{j} \text { for all } i \in \mathbf{S}_{+}, \\
y(0, j, i) & =a(0, j, i) \beta \min _{k \in S^{p}(i)}\left\{\Delta_{k}\right\} \text { for all }(i, j) \in \mathbf{S}_{+} \times \mathbf{S}_{+}, \text {and } \\
y(j, 0, i) & =\frac{\beta}{\gamma_{i}^{1}\left(\mathbf{S}_{+}\right)} \sum_{k=1}^{S} \pi_{i k}\left(\lambda-\theta_{k i}\right) \Delta_{k} \text { for all }(i, j) \in \mathbf{S}_{+} \times \mathbf{S}_{+} .
\end{aligned}
$$

Then define $f(\Delta) \equiv b(g(\Delta))+\beta C(a) \Delta$, where the vector $b$ and the matrix $C$ are as defined in (9.13). It follows that if $\Delta$ is a fixed point of $f$, then $g(\Delta)$ and the associated money transfers is a mechanism that satisfies (4.7)-(4.10) and vice versa. Therefore, for existence and uniqueness, the claims in the lemma, it is enough to show that $f(\Delta)$ satisfies conditions (i)-(v).

The mapping $g$ is increasing and $b$ is also increasing. Therefore, $f$ is increasing. Because the min function is concave, it follows that $g$ is concave. And because $b$ is concave in $y, b(g(\Delta))$ is the composition of two increasing concave functions, which is concave. Also, $f^{i}(\Delta)$ can be written in the form 


$$
f^{i}(\Delta)=\frac{1}{N} \sum_{j=1}^{S} \pi_{i j}\left(\lambda-\eta_{i j}\right) \theta_{j i} u(y(j, j, i))+\Psi(\Delta),
$$

where $\Psi$ is a nonnegative concave function, $\pi_{i j}\left(\lambda-\eta_{i j}\right) \theta_{j i}$ is positive, and $y(j, j, i)=$ $\frac{\Delta_{j}}{\beta}$. Because $u$ is strictly concave, this implies that $f^{i}$ is strictly concave.

Let $\Delta^{a}$ be the constant vector with $\Delta_{j}^{a}=\frac{\varepsilon}{\beta}, j \in \mathbf{S}_{+}$. Then

$$
f^{i}\left(\Delta^{a}\right) \geq \frac{u(\varepsilon)}{N} \sum_{j=1}^{S} \pi_{i j}\left(\lambda-\eta_{i j}\right) \theta_{j i} \geq \frac{u(\varepsilon)}{N} \pi_{i i} \lambda \theta_{i i}=\frac{u(\varepsilon)}{N} \pi_{i i} \lambda^{2}\left(1-m_{i}\right) .
$$

Because $u^{\prime}(0)=\infty$, the ratio $\frac{u(\varepsilon)}{\varepsilon}$ becomes arbitrarily large as $\varepsilon \rightarrow 0$. Choose $\varepsilon$ so that

$$
\frac{u(\varepsilon)}{\varepsilon}>\frac{N}{\beta \pi_{i i} \lambda^{2}\left(1-m_{i}\right)}
$$

Then

$$
f^{i}\left(\Delta^{a}\right)>\frac{\varepsilon}{\beta}=\Delta^{a} .
$$

Thus $\Delta^{a}$ satisfies assumption (iv) of the fixed point theorem.

Let $\Delta^{b}$ be the constant vector with $\Delta_{j}^{b}=\frac{y^{*}}{\beta}, j \in \mathbf{S}_{+}$. (Recall that $u^{\prime}\left(y^{*}\right)=1$.) Then $f^{i}\left(\Delta^{b}\right)<\frac{y^{*}}{\beta}$ by essentially the argument in the proof of lemma 5 . Therefore, $\Delta^{b}$ satisfies assumption (v) of the fixed point theorem.

Finally, because the mapping $f$ satisfies condition (iii) with equality, $f$ has a unique positive fixed point $\hat{\Delta}$.

Lemma 7. Let $S^{p}(i)$ for $i \in \mathbf{S}_{+}$be given. Let $\mathbf{P}=\left\{\Pi: \pi_{i i} \geq \alpha>0\right.$ for all $\left.i\right\}$. Then the unique monetary mechanism that satisfies (4.7)-(4.10) is continuous in $\Pi$ for $\Pi \in \mathbf{P}$.

Proof. Let $\Delta^{*}(\Pi)$ be the unique positive fixed point of $f(\Delta ; \Pi)$, where, for a given $\Pi \in \mathbf{P}, f$ is the mapping defined in the proof of lemma 6 . That is, $f(\Delta ; \Pi) \equiv$ $b(g(\Delta))+\beta C(a) \Delta$, where $g$ is defined by (9.33)-(9.36). We now restrict the domain of $f$ to $\Delta \in\left[\Delta^{a}, \Delta^{b}\right]$, where $\Delta^{b}$ is the bound given in lemma 6 and where $\Delta^{a}$ is given by (9.39), but with $\pi_{i i}$ in (9.38) replaced by $\alpha$. So defined, the interval $\left[\Delta^{a}, \Delta^{b}\right]$ does not depend on $\Pi$. For a given $\Delta, f$ is continuous in $\Pi$, because it is a composite of two functions. each of which is continuous in the components of $\Pi$. And with $\Delta$ limited to the compact domain $\left[\Delta^{a}, \Delta^{b}\right]$, it follows that $f$ is uniformly continuous in $\Pi$. That is, for any $\varepsilon>0$, there exists a $\delta_{1}>0$ such that if $\left\|\Pi_{1}-\Pi_{2}\right\|<\delta_{1}$, then $\left\|f\left(\Delta ; \Pi_{1}\right)-f\left(\Delta ; \Pi_{2}\right)\right\|<\frac{\varepsilon}{3}$ for all $\Delta$.

Let $\Pi_{n}$ be a sequence converging to $\Pi$. Because the sequence $\Delta^{*}\left(\Pi_{n}\right)$ lies in a compact set, it has a convergent subsequence $\Delta^{*}\left(\Pi_{k}\right)$. Let $\hat{\Delta}$ be the limit of this subsequence. Then there exists a $\delta_{2}>0$ such that $\left\|\Pi_{k}-\Pi\right\|<\delta_{2}$ implies $\| f\left(\Delta^{*}\left(\Pi_{k}\right) ; \Pi_{k}\right)-$ 
$\hat{\Delta} \|<\frac{\varepsilon}{3}$. Also, because $f$ is concave in $\Delta$, and, therefore, continuous in $\Delta$, there exists a $\delta_{3}>0$ such that $\left\|\Pi_{k}-\Pi\right\|<\delta_{3}$ implies $\left\|f\left(\Delta^{*}\left(\Pi_{k}\right) ; \Pi\right)-f(\hat{\Delta} ; \Pi)\right\|<\frac{\varepsilon}{3}$. And by uniform continuity in $\Pi, \| \Pi_{k}-\Pi||<\delta_{1}$ implies ||$f\left(\Delta^{*}\left(\Pi_{k}\right) ; \Pi_{k}\right)-f\left(\Delta^{*}\left(\Pi_{k}\right) ; \Pi\right) \|<\frac{\varepsilon}{3}$. Thus, if $k$ is such that $\left\|\Pi_{k}-\Pi\right\|<\min \left(\delta_{1}, \delta_{2}, \delta_{3}\right)$, then

$$
\begin{gathered}
\|f(\hat{\Delta} ; \Pi)-\hat{\Delta}\| \leq\left\|f(\hat{\Delta} ; \Pi)-f\left(\Delta^{*}\left(\Pi_{k}\right) ; \Pi\right)\right\|+ \\
\left\|f\left(\Delta^{*}\left(\Pi_{k}\right) ; \Pi\right)-f\left(\Delta^{*}\left(\Pi_{k}\right) ; \Pi_{k}\right)\right\|+\left\|f\left(\Delta^{*}\left(\Pi_{k}\right) ; \Pi_{k}\right)-\hat{\Delta}\right\|<\varepsilon .
\end{gathered}
$$

Because this holds for any $\varepsilon>0, f(\hat{\Delta} ; \Pi)=\hat{\Delta}$. And because $f(\hat{\Delta} ; \Pi)$ has a unique positive fixed point, it follows that $\hat{\Delta}=\Delta^{*}(\Pi)$. Finally, if that original sequence $\Delta^{*}\left(\Pi_{n}\right)$ does not converge to $\Delta^{*}(\Pi)$, then it must have a subsequence that is bounded away from $\Delta^{*}(\Pi)$. But that subsequence must in turn have a convergent subsequence. By the argument just given, the limit of that subsequence can only be $\Delta^{*}(\Pi)$. Thus, $\Delta^{*}(\Pi)$ is continuous.

Proposition 2. If the optimum satisfies $u\left(\beta \min _{s} \Delta_{s}\right) \geq y_{\max }^{1}$, then the maximized objective is continuous in $\Pi$ for $\Pi$ such that $\pi_{i j}$ is positive and $\pi_{i i}$ is bounded away from 0 .

Proof. For a given transition matrix $\Pi$, let $\Omega(\Pi)$ be the set of mechanisms identified in Lemma 6 . Also, let $\Omega^{*}(\Pi)$ be the subset of $\Omega(\Pi)$ that is incentive-feasible. Because the always-trade mechanism $\left(S^{p}(i) \equiv \mathbf{S}_{+}\right)$is incentive-feasible and is in $\Omega(\Pi), \Omega^{*}(\Pi)$ is not empty.

Let $\Pi_{n} \rightarrow \Pi^{0}$, and let $Z_{n}^{*}$ be the optimal welfare for $\Pi=\Pi_{n}$, with $Z_{n}^{*}=$ $\zeta\left(y\left(\Pi_{n}\right), a\left(\Pi_{n}\right)\right)$, where the function $\zeta$ is defined by equation 3.16 ; also let $Z_{0}^{*}$ be the optimal welfare for $\Pi=\Pi_{0}$, with $Z_{0}^{*}=\zeta\left(y\left(\Pi^{0}\right), a\left(\Pi^{0}\right)\right)$.

Suppose $\lim \sup _{n \rightarrow \infty} Z_{n}^{*}>Z_{0}^{*}$. Then there is a positive number $\varepsilon$ and a subsequence $Z_{n_{j}}^{*}$ such that $Z_{n_{j}}^{*}>Z_{0}^{*}+\varepsilon$ for all $j$, with $Z_{n_{j}}^{*}=\zeta\left(y\left(\Pi_{n_{j}}\right), a\left(\Pi_{n_{j}}\right)\right)$. Because the set of possible $a^{\prime} s$ is finite (there is a finite number of possible specifications of the set $S^{p}(i)$ for each $i \in \mathbf{S}_{+}$), there must be at least one $a$ that occurs infinitely often in this subsequence. Denote such an $a$ by $\hat{a}$. Then we have, $\zeta\left(\hat{y}\left(\Pi_{n_{j}}\right), \hat{a}\right)>Z_{0}^{*}+\varepsilon$ for all $j$, where $\hat{y}\left(\Pi_{n_{j}}\right)$ denotes the unique lemma 6 mechanism for $a=\hat{a}$. Then, by lemma $7, \zeta\left(\hat{y}\left(\Pi^{0}\right), \hat{a}\right)>Z_{0}^{*}$. Therefore, the optimality of $Z_{0}^{*}$ implies $\left(\hat{y}\left(\Pi^{0}\right), \hat{a}\right) \notin \Omega^{*}\left(\Pi^{0}\right)$. But since $\left(\hat{y}\left(\Pi_{n_{j}}\right), \hat{a}\right) \in \Omega^{*}\left(\Pi_{n_{j}}\right)$, we have $\Delta_{r}\left(\hat{y}\left(\Pi_{n_{j}}\right), \hat{a}\right) \leq \Delta_{s}\left(\hat{y}\left(\Pi_{n_{j}}\right), \hat{a}\right)$ for all $i, r, s$ such that $r \notin \hat{S}^{p}(i)$ and $s \in \hat{S}^{p}(i)$, where $\hat{S}^{p}(i)$ is the set $S^{p}(i)$ that corresponds to $\hat{a}$. Because, again by Lemma 7 , these inequalities are preserved in the limit as $\Pi_{n_{j}} \rightarrow \Pi^{0}$, we have $\left(\hat{y}\left(\Pi^{0}\right), \hat{a}\right) \in \Omega^{*}\left(\Pi^{0}\right)$, a contradiction. Therefore, $\lim \sup _{n \rightarrow \infty} Z_{n}^{*} \leq Z_{0}^{*}$.

Let $a=\tilde{a}$ be optimal at $\Pi=\Pi^{0}$. By part (iii) of Proposition 1 we know that, for all $i \in \mathbf{S}_{+}, r \notin \tilde{S}^{p}(i)$ and $s \in \tilde{S}^{p}(i)$ implies $\Delta_{r}\left(\tilde{y}\left(\Pi^{0}\right), \tilde{a}\right)<\Delta_{s}\left(\tilde{y}\left(\Pi^{0}\right), \tilde{a}\right)$, where $\tilde{y}(\Pi)$ denotes the unique lemma 6 mechanism for $a=\tilde{a}$. Lemma 7 then implies that $\left(\tilde{y}\left(\Pi_{n}\right), \tilde{a}\right) \in \Omega^{*}\left(\Pi_{n}\right)$ for $n$ sufficiently large. Thus $Z_{n}^{*} \geq \zeta\left(\tilde{y}\left(\Pi_{n}\right), \tilde{a}\right)$, which implies $\lim \inf _{n \rightarrow \infty} Z_{n}^{*} \geq Z_{0}^{*}$.

It follows that $\lim _{n \rightarrow \infty} Z_{n}^{*}=Z_{0}^{*}$.

Proposition 3. Let $\left\{\Pi_{k}\right\} \rightarrow I$ (the identity matrix) and be such that the corresponding sequence of stationary probability vectors $\left\{p_{k}\right\}$ converges to a strictly 
positive vector. Assume that the optimum for any $\Pi$ in the neighborhood of $\Pi=I$ satisfies $u\left(\beta \min _{s} \Delta_{s}\right) \geq y_{\max }^{1}$. There exists $K$ such that if $k>K$, then the optimum for $\Pi_{k}$ has no trade between informed producers and uninformed consumers whenever the current state, $j$, exceeds the previous state, $i$.

Proof. Let $\left\{\Pi_{k}\right\} \rightarrow I$ be given and let $\left\{p_{k}\right\} \rightarrow p^{\prime}$. For $\Pi=I$, define the function, $Z(I)$, to be $Z$ with $p=p^{\prime}$.

The maximum of $Z(I)$ over incentive-feasible $(y, a)$ is $y\left(\iota^{c} i, \iota^{p} i,, i\right)=y_{\max }^{i}$. (When $\Pi=I$, the maximum is obtained state by state and for each state, the result is given in [3]. Denote that maximum $Z^{*}(I)$.

Now let $S^{p}(i)^{\prime}=\{1,2, \ldots, i\}$ for each $i$. Also, let $y^{k}$ and $\Delta^{k}$ denote the unique monetary mechanism that satisfies (4.7)-(4.10) of proposition 1 when $S^{p}(i)=S^{p}(i)^{\prime}$ and $\Pi=\Pi_{k}$. Finally, let $y^{\infty}$ and $\Delta^{\infty}$ denote $y^{k}$ and $\Delta^{k}$ for $\Pi=I$. Then, $y^{\infty}\left(\iota^{c} i, \iota^{p} i,{ }^{\prime} i\right)=$ $y_{\max }^{i}$ and $\Delta_{i}^{\infty}=y_{\max }^{i} / \beta$. (This is verified by inserting these expressions into (4.7)-(4.10 ) and (9.13) and noticing that they hold.) Then, by lemma $7, \lim _{k \rightarrow \infty} y^{k}\left(\iota^{c} i, \iota^{p} i,, i\right)=$ $y_{\max }^{i}$ and $\lim _{k \rightarrow \infty} \Delta_{i}^{k}=y_{\max }^{i} / \beta$. That, in turn, implies that if $k$ is sufficiently large, then $y_{k}^{\prime}$ is incentive feasible. (This follows because the limit result implies that if $k$ is large, then $\Delta_{i}^{k}>\Delta_{i+1}^{k}$. Now let $Z^{*}(\Pi)$ denote the maximized objective. It follows that for any $\varepsilon>0$, there exists $K$ such that $k>K$ implies $Z^{*}\left(\Pi_{k}\right)>Z^{*}(I)-\varepsilon$. We now show that if $\Pi_{k}$ is close enough to $I$, then a mechanism which has trade when the current state $j$ and previous state $i$ satisfy $j>i$ gives a value of the objective that is less than $Z^{*}(I)-\varepsilon$, and, therefore, cannot be optimal.

If $(y, a)$ satisfies producer individual-rationality constraints, then

$$
\lim _{\Pi \rightarrow I} \Delta_{i}(y, a ; \Pi) \leq y_{\max }^{i} / \beta .
$$

This follows by taking the limit of $\Delta_{i}(y, a)$ as given by (9.13) and noting two things: only terms of the form $\lim y\left(\iota^{c} i, \iota^{p} i, i\right)$ and $\lim \left[a\left(\iota^{c} i, \iota^{p} i, i\right) \beta \Delta_{i}\right]$ appear and the individualrationality constraints imply $\lim y\left(\iota^{c} i, \iota^{p} i, i\right) \leq \lim \left[a\left(\iota^{c} i, \iota^{p} i, i\right) \beta \Delta_{i}\right]$. Now suppose by contradiction that the no-trade claim is false. Then there exists a subsequence of the given sequence $\left\{\Pi_{k}\right\}$, call it $\left\{\Pi_{n}\right\}$, such that $\left\{\Pi_{n}\right\} \rightarrow I$ and for each $n$ the optimum has trade in some current state $j$, where $j>i$, the previous state. Let $Z^{\prime \prime}\left(\Pi_{n}\right)$ denote the implied value of $Z$. The producer truth-telling constraint and (9.41) imply that for sufficiently large $n$, either $a(0, i, i)^{n}=0$ or $y(0, i, i)^{n} \leq y_{\max }^{j}+\varepsilon_{0}$, where $j>i$ and where $\varepsilon_{0}>0$ but is otherwise arbitrary. (Recall that $y_{\max }^{j}<y_{\max }^{i}$ if $j>i$ and that $y_{\max }^{i}$ depends on $m_{i}$, but not on П.) But (9.41) also implies that for sufficiently large $n, y\left(\iota^{c} i, \iota^{p} i, i\right)^{n} \leq y_{\max }^{i}+\varepsilon_{i}$, where, again, $\varepsilon_{i}>0$ but is otherwise arbitrary. Because a component of $y$ appears with a non-vanishing coefficient in $\lim _{n \rightarrow \infty} Z\left(\Pi_{n}\right)$ if and only if it has the form $y\left(\iota^{c} i, \iota^{p} i, i\right)$, it follows that $\lim \sup \left\{Z^{\prime \prime}\left(\Pi_{k}\right)\right\}<Z^{*}(I)$, a contradiction.

Lemma 8. For each $k \in \mathbf{S}_{+}$, let $\Delta^{k} \in R^{S}$ be defined by $\Delta_{j}^{k}=y_{\max }^{1} / \beta$ for $j \neq k$ and $\beta \Delta_{k}^{k}=u^{-1}\left(y_{\max }^{1}\right)$. Then let $y^{k}$ be defined by

$$
y^{k}(j, 0, i)=\frac{1}{\gamma_{i}^{1}\left(\mathbf{S}_{+}\right)} \sum_{s=1}^{S} \pi_{i s}\left(\lambda-\theta_{s i}\right) \beta \Delta_{s}^{k}
$$




$$
y^{k}(j, j, i)=y^{k}(0, j, i)=\beta \Delta_{j}^{k}, y^{k}(0,0, i)=\beta \sum_{s=1}^{S} \pi_{i s} \Delta_{s}^{k},
$$

for all $i, j \in \mathbf{S}_{+}$. Also, let $Z\left(y^{k}\right)$ be ex ante welfare implied by $y^{k}$ evaluated from (3.16). Let $Z_{\min }$ be the ex ante welfare implied by some incentive-feasible mechanism. If there exists $Z_{\text {min }}$ such that $Z\left(y^{k}\right) \leq Z_{\text {min }}$ for each $k \in \mathbf{S}_{+}$, then any optimum satisfies $u\left(\beta \min _{s} \Delta_{s}\right) \geq y_{\max }^{1}$.

Proof. If the optimum violates $u\left(\beta \min _{s} \Delta_{s}\right) \geq y_{\max }^{1}$, then for some $k \in \mathbf{S}_{+}$, $\beta \Delta_{k}<u^{-1}\left(y_{\max }^{1}\right)$. (Notice that $u^{-1}\left(y_{\max }^{1}\right)<y_{\max }^{1}$.) If so, then $y^{k}$ is strictly greater than any optimal $y$. For $y^{k}(j, 0, i)$, this follows from lemma 3 . For $y^{k}(j, j, i)$ and $y^{k}(0,0, i)$, it follows directly from the respective producer individual rationality constraints. Finally, for $y^{k}(0, j, i)$, consider the second inequality in (3.11), which is the individual rationality constraint for informed producers. If $j \in S^{p}(i)$, then the minimum is no greater than $\beta \Delta_{j}^{k}$. If $j \notin S^{p}(i)$, then $y(0, j, i)=0$. In either case, we get $y(0, j, i) \leq y^{k}(0, j, i)$. Therefore, $Z\left(y^{k}\right)$ is strictly greater than the optimum $Z$. But if $Z\left(y^{k}\right) \leq Z_{\min }$ for each $k \in \mathbf{S}_{+}$, then we have a contradiction and the optimum cannot satisfy $u\left(\beta \min _{s} \Delta_{s}\right)<y_{\max }^{1}$.

Lemma 9. There exists $\varepsilon>0$ such that if $m_{S}-m_{1}<\varepsilon$, then the hypothesis of lemma 8 holds.

Proof. Let $\left(y_{x}, 1\right)$ denote an always-trade mechanism with $y_{x}\left(s, s^{\prime}, i\right) \equiv x$, a mechanism in which trade occurs in every trade meeting and output is the same in every trade meeting. For such a mechanism,

$$
c_{i j}(a)=\left(1-\frac{1}{N}\right) \pi_{i j}\left(1-\eta_{i j}\right),
$$

and

$$
\begin{gathered}
N b_{i}\left(y_{x}\right)=(1-\lambda) \sum_{j} \pi_{i j}\left[\left(1-m_{j}\right) u(x)+m_{j} x\right]+ \\
\sum_{j} \pi_{i j}\left(\lambda-\eta_{i j}\right)\left[\left(1-m_{j}\right) u(x)+m_{j} x\right]= \\
\sum_{j} \pi_{i j}\left(1-\eta_{i j}\right)\left[\left(1-m_{j}\right) u(x)+m_{j} x\right],
\end{gathered}
$$

where the first equality in (9.44) comes from using the definition of $\theta_{j i}$ to combine, in turn, the first two lines and the last two lines of the expression for $N b_{i}(y)$ (see (4.6)). If $u(x) \geq x$, which is implied by $x \leq y_{\max }^{1}$, then

$$
N b_{i}\left(y_{x}\right) \geq\left[\left(1-m_{S}\right) u(x)+m_{S} x\right] \Phi(i)
$$

where $\Phi(i) \equiv \sum_{j} \pi_{i j}\left(1-\eta_{i j}\right)$. Now let $L=\min _{i} \Delta_{i}\left(y_{x}, 1\right)$. Then by (9.13), $L$ satisfies

$$
N L \geq N \min _{i} b_{i}\left(y_{x}\right)+(N-1) \beta L \min _{i} \Phi(i) .
$$


Therefore, by (9.45), $L$ satisfies

$$
N L \geq\left[\left(1-m_{S}\right) u(x)+m_{S} x\right] \Phi+(N-1) \beta L \Phi
$$

where $\Phi \equiv \min _{i} \Phi(i)$. Suppose, then, that we take for $x$ the positive solution to $x=\beta L$ and (9.47) at equality — namely, the unique positive solution for $x$ to

$$
x=\frac{\beta \Phi z(x)\left(1-m_{S}\right)}{(1-\beta \Phi) N} .
$$

(Notice that this solution is less than $y_{\max }^{S}$ because $\Phi<1$. In fact, it is $y_{\max }^{S}$ for an economy with a discount factor $\beta^{\prime}=\beta \Phi<\beta$.) By construction, then, $\left(y_{x}, 1\right)$ with $x$ given by the positive solution to (9.48) satisfies producer individual-rationality constraints. We next have to satisfy consumer individual-rationality constraints.

Let $K=\max _{i} \Delta_{i}\left(y_{x}, 1\right)$. Then by $(9.13), K$ satisfies

$$
N K \leq N \max _{i} b_{i}\left(y_{x}\right)+(N-1) \beta K \max _{i} \Phi(i) .
$$

Therefore, by the same reasoning that led to (9.45), $K$ satisfies

$$
N K \leq\left[\left(1-m_{1}\right) u(x)+m_{1} x\right]+(N-1) \beta K,
$$

where we have taken unity to be an upper bound on $\Phi(i)$. With $x$ given by the positive solution to (9.48), it follows that $K \leq y_{\max }^{1} / \beta$. Therefore, it is sufficient for satisfaction of consumer individual-rationality constraints for the mechanism $\left(y_{x}, 1\right)$ with $x$ given by the positive solution to $(9.48)$ that $u(x) \geq y_{\max }^{1}$.

Because truth-telling is vacuous for the mechanism $\left(y_{x}, 1\right)$, that mechanism is incentive-feasible provided $u(x) \geq y_{\max }^{1}$, which is a condition that is easy to check. If it holds, then $Z\left(y_{x}\right)$ is a possible magnitude for $Z_{\min }$.

To complete the proof, we first show that $x \rightarrow y_{\max }^{1}$ as $m_{S}-m_{1} \rightarrow 0$. From (3.5) it follows that $\eta_{i j} \rightarrow 0$ as $m_{S}-m_{1} \rightarrow 0$. Therefore, $\Phi(i) \rightarrow 1$ as $m_{S}-m_{1} \rightarrow 0$. It follows that as $m_{S}-m_{1} \rightarrow 0, \Phi \rightarrow 1$ and, hence, by the definition of $x$ that $x \rightarrow y_{\max }^{1}$. That is, as $\Phi \rightarrow 1$ and $m_{S} \rightarrow m_{1}$ in (9.48), then the unique positive solution to (9.48) approaches the unique positive solution to (4.1) for $m_{i}=m_{1}$. Now, for $x$ sufficiently close to $y_{\max }^{1}$, we have $u(x) \geq y_{\max }^{1}$. Finally, for $x$ sufficiently close to $y_{\max }^{1}$, it follows that the hypothesis of lemma 8 holds. 


\section{References}

[1] R.J. Barro and R.G. King, Time-separable preferences and intertemporalsubstitution models of business cycles. Quarterly Journal of Economics, 99 (1984) 817-839.

[2] A. Berentsen, M. Molico, and R. Wright, Indivisibilities, lotteries, and monetary exchange, Manuscript,University of Pennsylvania, December 1998.

[3] R. Cavalcanti and N. Wallace. "A model of private banknote issue." Review of Economic Dynamics 2 (February, 1999), 104-136.

[4] B. Eden, The adjustment of prices to monetary shocks when trade is uncertain and sequential. Journal of Political Economy, 102 (1994) 493-509.

[5] D. Hume, On money. In Writings on Economics. Edited by E. Rotwein. (Madison, University of Wisconsin Press. 1970)

[6] L. Jones and R. Manuelli, Volatile policy and private information: the case of monetary policy. NBER Working paper 7072, May 1999.

[7] J. Kennan, An elementary fixed point theorem for increasing concave functions on $\mathbb{R}^{n}$. http://www.ssc.wisc.edu/ $\sim$ jkennan/research/fixedpoint.pdf., 1999.

[8] J. Kennan and R. Wilson, Bargaining with private information. Journal of Economic Literature, 31 (1993), 45-104.

[9] N. Kocherlakota, Money is memory. Journal of Economic Theory, 81 (1998) 232251.

[10] R.E. Lucas Jr., Expectations and the neutrality of money. Journal of Economic Theory, 4 (1972) 103-124.

[11] R.E. Lucas Jr. and M. Woodford, Real effects of monetary shocks in an economy with sequential purchases. 1994, University of Chicago working paper.

[12] R. Myerson and M. Satterthwaite, Efficient mechanisms for bilateral trading. Journal of Economic Theory, 28 (1983), 265-281.

[13] S. Shi, Money and prices: a model of search and bargaining. Journal of Economic Theory, 67 (1995) 467-498.

[14] A. Trejos and R. Wright, Search, bargaining, money and prices. Journal of Political Economy, 103 (1995) 118-141.

[15] N. Wallace, Short-run and long-run effects of changes in money in a randommatching model. Journal of Political Economy, 105 (1997), 1293-1307.

[16] N. Wallace, Introduction to modeling money and studying monetary policy, Journal of Economic Theory, 81 (August 1998), 223-231. 